\title{
Postural Synergy-Based Exoskeleton Reproducing Natural Human Movements to Improve Upper Limb Motor Control after Stroke
}

\section{Chang He}

Huazhong University of Science and Technology - Main Campus: Huazhong University of Science and Technology

Cai-Hua Xiong ( $\nabla$ chxiong@hust.edu.cn )

Huazhong University of Science and Technology https://orcid.org/0000-0003-2326-0289

\section{Ze-Jian Chen}

Tongji Hospital of Tongji Medical College of Huazhong University of Science and Technology

\section{Wei Fan}

Tongji Hospital of Tongji Medical College of Huazhong University of Science and Technology

\section{Xiao-Lin Huang}

Tongji Hospital of Tongji Medical College of Huazhong University of Science and Technology

\section{Research}

Keywords: Postural synergy, Stroke, Rehabilitation, Exoskeleton, Upper limbs

Posted Date: November 13th, 2020

DOI: https://doi.org/10.21203/rs.3.rs-104427/v1

License: (c) (i) This work is licensed under a Creative Commons Attribution 4.0 International License. Read Full License 


\title{
Postural Synergy-Based Exoskeleton Reproducing Natural Human Movements
} to Improve Upper Limb Motor Control after Stroke

\author{
Chang He ${ }^{1}$ Cai-Hua Xiong ${ }^{1 *}$ Ze-Jian Chen ${ }^{2}$ Wei Fan ${ }^{2}$ Xiao-lin Huang ${ }^{2}$ \\ ${ }^{1}$ State Key Lab of Digital Manufacturing Equipment and Technology \\ School of Mechanical Science and Engineering \\ Huazhong University of Science and Technology, Wuhan 430074, Hubei, China. \\ chxiong@hust.edu.cn \\ ${ }^{2}$ Department of Rehabilitation Medicine \\ Tongji Hospital, Tongji Medical College \\ Huazhong University of Science and Technology, Wuhan 430030, Hubei, China.
}

\section{ABSTRACT}

Background: Upper limb exoskeletons have drawn significant attention in neurorehabilitation because of anthropomorphic mechanical structure analogous to human anatomy. Whereas, the training movements are typically underorganized because most exoskeletons only control the movement of the hand in space, without considering rehabilitation of joint motion, particularly inter-joint postural synergy. The purposes of this study were to explore the application of a postural synergy-based exoskeleton (Armule) reproducing natural human movements for robot-assisted neurorehabilitation and to preliminarily assess its effect on patients' upper limb motor control after stroke.

Methods: We developed a novel upper limb exoskeleton based on the concept of postural synergy, which provided five degrees of freedom (DOF), natural human movements of the upper limb. Eight participants with hemiplegia due to a first-ever, 
unilateral stroke were recruited and included. They participated in exoskeleton therapy sessions 45 minutes/day, 5 days/week for 4 weeks, with passive/active training under anthropomorphic trajectories and postures. The primary outcome was the Fugl-Meyer Assessment for Upper Extremities (FMA-UE). The secondary outcomes were the Action Research Arm Test(ARAT), modified Barthel Index (mBI), and exoskeleton kinematic as well as interaction force metrics: motion smoothness in the joint space, postural synergy error, interaction force smoothness, and the intent response rate.

Results: After the 4-weeks intervention, all subjects showed significant improvements in the following clinical measures: the FMA-UE $(p=0.02)$, the ARAT $(p=0.003)$, and the $\mathrm{mBI}$ score $(p<0.001)$. Besides, all subjects showed significant improvements in motion smoothness $(p=0.004)$, postural synergy error $(p=0.014)$, interaction force smoothness $(p=0.004)$, and the intent response rate $(p=0.008)$.

Conclusions: The subjects were well adapted to our device that assisted in completing functional movements with natural human movement characteristics. The results of the preliminary clinical intervention indicate that the Armule exoskeleton improves individuals' motor control and activities of daily living (ADL) function after stroke, which might be associated with kinematic and interaction force optimization and postural synergy modification during functional tasks.

Clinical trial registration: ChiCTR, ChiCTR1900026656; Date of registration: October 17, 2019. http://www.chictr.org.cn/showproj.aspx?proj=44420

Keywords: Postural synergy, Stroke, Rehabilitation, Exoskeleton, Upper limbs 


\section{Background}

Stroke is the leading cause of adult mortality and disability worldwide[1], with consequent upper extremity motor dysfunction severely affecting the quality of life in stroke survivors and bringing great domestic and socioeconomic burden. Motor relearning theories are often used to guide stroke rehabilitation[2], which requires training of movement components and integrated functional practice[3] under the mechanism of use-dependent plasticity[4] and operant reinforcement processes[5]. Systematic reviews have also suggested that patients benefit from task-specific training directly rather than from impairment-oriented interventions[6-8].

Robot-assisted training is an innovative exercise-based therapy that involves the principles of motor learning, and it can provide highly intensive, adaptive, and taskspecific training as well as feedback and motivation for enhancing neuroplasticity. Over the last decades, various robotic devices with different training modalities, structures, and principles have been developed for upper-limb training after stroke, but the benefits compared with traditional methods are controversial in terms of functional performance, especially in movement quality and independence in ADL[9]. Therefore, how to optimize the design and training methods of upper limb robots to relearn natural human movements in $\mathrm{ADL}[10]$ remains important for researchers to determine.

Owing to the multiple attachments to the patient's limb, exoskeleton rehabilitation robots can assist patients in accomplishing complex functional movements in threedimensional space[11]. Most upper limb exoskeletons have more than five DOF for 
shoulder, elbow, and wrist movements so that they can adapt to the human upper limb[12], which has superior motor control; some examples include CADEN-7[13], ARMin-V[14], Harmony[15], MGA[16], LIMPACT[17] and ANYexo[18]. Although exoskeleton robots provide many functions for motion, such mechanical structures can pose challenges for the control strategy[19]. To complete specific training tasks, especially ADL tasks, most robots need to generate a joint reference trajectory in a high dimensional space[11] to drive the robot directly or serve as a reference for other control strategies. The joint reference trajectories can be exploited from the recordings of movements in healthy subjects or informed by a therapist, but the types of training tasks are limited[20]. The joint reference trajectories can also be computed by an optimal trajectory planner or other motion planners. However, the laws of human upper limb movement are still unclear[21], and the safety of the trajectory generated by the optimization method or other motion planners is questionable; hence, the reference trajectory generated by these methods does not fully reproduce the natural human movements.

To encourage patients to participate in exoskeleton training actively, some exoskeleton robots use electromyography [22], electroencephalograph [23], and other electrophysiological tools[24] to drive the rehabilitation robot according to the patient's own intention. Nevertheless, the process of decoding electrophysiological signals is exceptionally complicated, with a low success rate and accuracy due to the highdimensional input and output signals[25]. As a result, most exoskeleton robots adopt control strategies based on human-robot physical interactions[26], such as assist-as- 
needed, impedance control, and admittance control. All of these strategies should improve the transparency of control to the greatest extent possible[27]; that is, the robot must interfere with the patient's autonomous movement as little as possible. Although robots with high transparency can encourage the patient to participate in the training actively[28], they amplify the abnormality of patients' postures and compensatory movements[29]. High transparency also increases the complexity of the structure and control of the exoskeleton[30].

Bernstein's theory of motor control suggested that human motions are quite stereotyped and that motor synergy patterns are common among all humans[31]. Synergy is characterized by the relationship between kinematics, dynamics, or other physiological parameters sharing the same spatial-temporal properties[32]. According to this theory, if the synergies of the human upper limb for specific tasks can be identified, they can be used to reconstruct the natural human movement of the upper limb[33]. Several pilot studies have employed synergies at the muscle level to evaluate the changes in muscle synergies after stroke[34, 35] and included synergies as outcome measures to evaluate stroke recovery[36, 37]. Kinematic synergy, or postural synergy, can be directly used in the reconstruction of natural human movement in applications such as the design of artificial limbs[33] or rehabilitation robots[38-40] due to its intuitive performance. Some recent studies have shown that postural synergies can improve the efficiency of motor learning in healthy people[41-43] and that these synergies show great potential in the field of rehabilitation. To the authors' knowledge, currently, no synergy-based rehabilitation exoskeletons are used in clinical practice. 
113 Aiming to meet the above clinical needs for rehabilitation exoskeletons and overcome

114 the existing problems related to rehabilitation exoskeletons, we developed a postural

115 synergy-based rehabilitation exoskeleton[38] with an active training control strategy

116 using an interactive force to guide the rehabilitation exoskeleton movements in a

117 synergy dimension-reduced subspace. The preliminary evaluation and tests are 118 described in this paper.

\section{Methods}

\section{Equipment structure}

In previous work[38], we proposed the design of a rehabilitation exoskeleton based on postural synergy. Natural self-reaching movements of the human arm were analyzed by principal component analysis (PCA). The most significant synergies of the human arm joints, which accounted for more than $80 \%$ of the variation, were extracted. Then, postural synergies were used to guide the design of the kinematic transmission mechanism.

$$
\boldsymbol{\theta}=\overline{\boldsymbol{\theta}}+\boldsymbol{C u}
$$

129 where $\boldsymbol{\theta}=\left[\begin{array}{ll}\theta_{1} \ldots \theta_{5}\end{array}\right]^{\mathrm{T}}$ and $\boldsymbol{u}=\left[\begin{array}{ll}u_{1} & u_{2}\end{array}\right]^{\mathrm{T}}, \overline{\boldsymbol{\theta}}$ is the biased vector from the initial 130 joint angle and $\boldsymbol{C}$ is the coupling matrix. Several kinds of ADL self-reaching 131 movement data were collected, and PCA was used to extract the moving principal 132 components to obtain $\boldsymbol{C}$. That is, $\boldsymbol{C}$ contains the natural human movement 133 characteristics of these ADL movements. 
Based on this idea, we developed the Armule rehabilitation exoskeletal robot, as shown in Figure 1, which has 5 DOF driven by two actuators and the attached coupling kinematic transmission mechanism. The mechanical structure design is embedded with the postural synergies of functional movements, enabling the robot to reconstruct natural human reaching movements inherently; in other words, the exoskeleton produces movements that are humanoid.

Several changes relative to the previous mechanical structure were implemented in this work. In previous work, the patient's subjective motion intention was estimated through a six-dimensional force/torque sensor fixed to the end of the exoskeleton's handle and converted into a commanded velocity in the two-dimensional manifold of the driving space with a certain algorithm. Hence, the robot can generate assistive movements similar to natural human movements in response to the user's motion intentions. However, patients in the early stage of a rehabilitation treatment are typically unable to move their distal joints to apply high enough interaction forces to the handle to trigger the sensor. Therefore, we removed the handle and updated the mechanical design of the linkage cuffs. The wrist joints were instead fixed in the forearm linkage cuff. Pressure sensors and six-dimensional force/torque sensors were installed in the linkage cuffs of the forearm and upper arm, respectively, to estimate the patient's motion intention.

\section{Control strategy}

To generate assistive movements similar to natural reaching movements in response to the user's motion intentions, in previous work[44], a Riemannian metric was proposed 
to generate anthropomorphic reaching movements according to contact forces and torques. We detected the interaction forces between the patient and the robot handle to determine the patient's subjective movement intention. The interaction forces were converted to the command speed in the work space with an admittance control method. The actuator velocities were then obtained by their projection in the two-dimensional manifold in the drive space.

Because of the mechanical structure changes, the way we identified intentions changed correspondingly, and an admittance control method in the joint space was developed. Unlike the previous approach, the interaction forces between the patient and robot were detected with a six-dimensional force/torque sensor and pressure sensor placed in the linkage cuffs along the upper arm and forearm. The Newton Euler equation was used to calculate the equivalent interaction force at the end of the rehabilitation robot, which was taken as the patient's motion intention.

Once we acquired the patient's intention to move, we used the admittance control method in the joint space to translate it into the joint command velocity. Finally, the joint command velocity was projected into the drive space.

$$
\begin{gathered}
\boldsymbol{V}_{D}=\boldsymbol{P} \boldsymbol{A} \boldsymbol{J}\left({ }_{u}^{T} \boldsymbol{T} \boldsymbol{F}^{u}+{ }_{f}^{T} \boldsymbol{T} \boldsymbol{F}^{f}\right) \\
\boldsymbol{P}=\left(\boldsymbol{C}^{T} \boldsymbol{C}\right)^{-1} \boldsymbol{C}^{T}
\end{gathered}
$$

$\boldsymbol{F}^{f}=\mathbf{0}$ when only using the upper arm sensor. Where $\boldsymbol{V}_{D}$ is velocity vector in drive space, $\boldsymbol{A}$ is admittance matrix, $\boldsymbol{J}$ is Jacobian matrix of exoskeleton, ${ }_{u}^{T} \boldsymbol{T}{ }_{f}^{T} \boldsymbol{T}$ are the adjoin matrices of the upper arm and forearm to the end of the exoskeleton respectively. $\boldsymbol{F}^{u} \boldsymbol{F}^{f}$ are the force vectors detected in the upper arm and forearm 

respectively. $\boldsymbol{P}$ is the projection matrix.

178 Under such a control strategy, the exoskeleton can drive the arm and the exoskeleton 179 according to the patient's perceived subjective movement intention.

180 Since the rehabilitation robot can only produce anthropoid motions derived from the

181 linear combination of synergy primitives, the calculated movement intention of the 182 patient might be inconsistent with the actual movement direction of the robot. In general, 183 the magnitude of the deviation is related to the operating point and the direction of the applied force, which is determined by the subspace projection formula (as shown in

185 Figure 2).

The rehabilitation robot can be easily driven only when the patient's movement intention is similar to the human natural movement characteristics. We believe that this kind of control mode is not entirely transparent, which can help suppress abnormal movement patterns and enable patients to relearn regular coordination without causing severe discomfort.

\section{Weight compensation}

The exoskeleton in this study adopts both a mechanical structure that provided weight compensation and a dynamic-based weight compensation algorithm to offset the 194 gravity of patient's and the robotic arm. Thus, the patient's ability to generate residual motion can be improved, enabling the training of active reaching movements. When in operation, the mechanical weight compensation structure applies the appropriate 
torques to the actuated joints to balance the arm's weight and reduce the burden of the motor unit. On the other hand, the exoskeleton compensates for the patient's arm weight in the control system using a dynamic model-based approach, and six-dimensional force/torque sensor data is used to estimate the patient's arm weight when the patient is in the passive training mode and compensate during the active training mode to eliminate the influence of the patient's arm weight on the process of determining the user's motion intention. The compensation coefficient can be adjusted in the humancomputer interaction interface.

\section{Rehabilitation training application}

The rehabilitation training program consists of two phases. In the first phase, the passive mode, the rehabilitation exoskeleton guides the patient to perform natural reaching movements designated and recorded by therapists. In the second phase, the rehabilitation robot is in the active mode, with a six-dimensional force/torque sensor in the upper arm and a pressure sensor in the forearm. The exoskeleton calculates and assists the patient in performing the natural reaching movement closest to the patient's intention based on the motion tendencies of the whole arm. In this phase, the robotic exoskeleton detects patients' intentions and assists patients in achieving anthropomorphic movements as well as ADL-related tasks with virtual games, such as shooting targets, playing whack-a-mole, drinking water, wiping the face, cleaning a window, and frying eggs (Figure $3 \mathrm{~A}, \mathrm{~B}$ ). The rotation angles of the two active joints are associated with the position of the operating objects in the virtual environment, with a 
fixed mapping relationship. Two active synergy primitives drive the exoskeleton, and

219 the patient can see the corresponding information on a screen. The exoskeleton guides

220 patients with visual cues and provides feedback to complete tasks involving

221 anthropomorphic postures and trajectories.

Participants

In order to test the feasibility of the exoskeleton for upper limb rehabilitation, 8 participants (age, $47.0 \pm 9.9$ years; time since stroke, $70.1 \pm 47.7$ days; see Table 1 )

225 were enrolled in the study. The inclusion criteria were as follows: (1) an age of 18-80

226 years; (2) a clinical diagnosis of the first-ever stroke within 6 months before enrollment;

227 and (3) upper limb hemiplegia, defined as a score between 8 and 47 on the FMA-UE.

228 The exclusion criteria were as follows: (1) an orthopedic condition of the upper limb,

229 e.g., fixed contracture, shoulder subluxation, severe arthritis, or recent fracture; and (2)

230 severe cognitive defects or aphasia hindering the patient's ability to understand or

231 follow instructions.

232 Table 1 Participant Characteristics

\begin{tabular}{ccccccccc}
\hline $\begin{array}{c}\text { Subjects } \\
\text { ID }\end{array}$ & Gender & $\begin{array}{c}\text { Age } \\
\text { (years) }\end{array}$ & $\begin{array}{c}\text { Days } \\
\text { between } \\
\text { onset and } \\
\text { enrollment }\end{array}$ & $\begin{array}{c}\text { Stroke } \\
\text { type }\end{array}$ & NIHSS & $\begin{array}{c}\text { Paretic } \\
\text { side }\end{array}$ & $\begin{array}{c}\text { FMA- } \\
\text { UE }\end{array}$ & MMSE \\
\hline 1 & M & 43 & 100 & H & 5 & L & 25 & 24 \\
2 & M & 65 & 97 & I & 2 & L & 32 & 30 \\
3 & M & 45 & 152 & H & 4 & L & 36 & 27 \\
4 & M & 38 & 94 & H & 6 & R & 9 & 25 \\
5 & M & 48 & 16 & H & 7 & L & 8 & 29 \\
6 & M & 33 & 42 & I & 2 & R & 60 & 30 \\
7 & M & 49 & 24 & I & 6 & L & 21 & 25 \\
8 & M & 55 & 36 & I & 6 & L & 8 & 24 \\
\hline
\end{tabular}




\section{Experimental protocol}

Demographic information, stroke type, time since stroke onset, mini-mental state examination (MMSE) scores were collected at baseline. The clinical outcome measures were assessed at baseline and immediately after the 4-week intervention. To avoid assessment bias, an independent evaluator blinded to the study procedure completed all the outcome measures. The participants were asked to sit in a chair with a backrest in front of the exoskeleton, and their torsos were secured with chest straps to prevent compensatory movements. The upper limbs were initially held in a natural, relaxed position. The exoskeleton attachments were adjusted according to the length and circumference of each patient's upper limb.

The participants received task-specific training involving 5-DOF movements of the upper extremity in a 3-dimensional workspace assisted by the exoskeleton. Therapy was delivered at the same frequency and duration: 45 minutes daily, 5 days/week for 4 weeks (total of 20 sessions). The participants were asked to perform two sets of exercises per session. Each set consisted of 5 minutes of passive training and 10 minutes of active training (Figure 3C). To eliminate the interference of different tasks, the patients were asked to complete five reaching movement tasks (frying eggs) in a virtual reality environment for each active training phase. For this training task, they put a virtual raw egg into a pan on the screen. The participants were verbally encouraged to do their best to complete the task at their preferred speed. 
The primary outcome was the FMA-UE score, which reflected the severity of the participants' upper extremity motor impairments. The secondary outcomes were the ARAT score, which reflected upper limbs performance, especially the hand's fine motor function, and the MBI score, which reflected the patient's level of functional independence in ADL. In addition, we designed two 10-point Likert scales (LSs), measuring the subjects' perception of their level of enjoyment and degree of improvement[45] (see supplementary material).

\section{Measurements with the exoskeleton}

Throughout the training period, the sensors on the exoskeleton monitored the kinematics and interactive forces, which were sampled at 100 Hertz and stored in text format. Then, the data were extracted through a semiautomatic custom program in MATLAB software (version 2018A, The MathWorks, Natick, Massachusetts, USA.) to analyze the kinematics and human-computer interaction indicators. In this paper, we used four indicators measured by the robot to evaluate the motion control ability of the patients with the assistance of the robot. We previously recorded exercise data from 10 healthy individuals performing the same task using the exoskeleton for reference.

272 Motion smoothness in the workspace $S_{w}$ : This parameter indicates whether the

273 patient's joint trajectories are mixed with other submovements. The extent of $S_{w}$ 274 during the reaching movement was characterized by path jerk, the third derivative of 275 the joint angle of the patients. The smaller the jerk was, the smoother the trajectory. 


$$
S_{w}=\frac{1}{T} \sum_{t=1}^{T}\|\dddot{s}\|_{F}
$$

Where $s$ is the position of the end of the exoskeleton in workspace, $T$ is the normalized time parameter

Postural synergy error $P_{e}$ : This measure indicates the deviation between the actual attitude and the anthropomorphic attitude in the whole arrival trajectory. It is defined as the covariance of the distance between the patient's trajectories and the healthy person in two synergy basis directions when the subjects performed the same training tasks. According to uncontrolled manifold theory, humans have a capacity for redundant motions to execute a given movement with flexibility and robustness. The coefficient of error was high at the segments with low covariance in the direction of the synergy basis to prevent high postural deviations. In contrast, the coefficient of the deviation was low at the segments with high covariance in the direction of the synergy basis to allow large postural variations.

$$
P_{e}=\frac{1}{T} \sum_{t=1}^{T} \sqrt{\left(\boldsymbol{S}_{t}-\overline{\boldsymbol{\mu}}_{t}\right)^{T} \boldsymbol{\Sigma}_{t}^{-1}\left(\boldsymbol{S}_{t}-\overline{\boldsymbol{\mu}}_{t}\right)}
$$

Where $\boldsymbol{S}_{t}$ is the active joint position at $t, \overline{\boldsymbol{\mu}}_{t}$ is the mean active joint positions for healthy people at $t, \boldsymbol{\Sigma}_{t}$ is the covariance matrix of active joint position of healthy people at $t$.

Interaction force smoothness $S_{f}$ : This measure indicates whether the interaction force between the patient and the exoskeleton is insufficient or excessive during the completion of the task. Both the excessive and insufficient representation of the 
interaction force accounted for deviations from the natural movement. The extent of $S_{f}$ during the reaching movement was characterized by the jerk interaction force, the gradient of the interaction force. The smaller the jerk was, the smoother the interaction force.

$$
S_{f}=\frac{1}{n T} \sum_{t=1}^{T} \sum_{i=1}^{n} \ddot{F}^{2}
$$

Where $n=6$.

Intent response rate $\boldsymbol{R}_{\boldsymbol{i}}$ : This parameter was defined as the cosine of the angle between the direction of the subject's intention to move, i.e., the direction of equivalent end interaction force, and the actual direction in which the exoskeleton moved. This measure reflects the extent to which the subject's movement intentions are executed by the exoskeleton.

$$
R_{i}=\cos (\theta)=\frac{\boldsymbol{F} \cdot \boldsymbol{V}}{\|\boldsymbol{F}\| \cdot\|\boldsymbol{V}\|}
$$

According to the definition, $\mathrm{R}_{\mathrm{i}} \in(0,1)$. The closer to zero $\mathbf{R}_{\mathbf{i}}$ is, the more exoskeleton assists the participant. The closer to $1 \mathbf{R}_{\mathbf{i}}$ is, the more similar the movement of the exoskeleton is to the patient's movement intention.

\section{Statistical analysis}


samples $t$-tests (for continuous variables) and Fisher's exact tests (for categorical

319 variables). Before and after the intervention, differences were compared using paired ttests (for normally distributed data) and Wilcoxon signed-rank tests (for nonparametric

321 equivalent tests). For all the statistical tests conducted, a two-sided $p$-value of less than 3220.05 was considered significant.

\section{Results}

\section{Exoskeleton kinematic and interaction force metrics}

Figure 4 shows two exemplar subjects' performance in the reaching tasks pretreatment and post-treatment. The patients showed significant improvements in motion smoothness in the joint space (difference, $7.22 \pm 2.55 ; p=0.004$ ), postural synergy error (difference, 107.54 $\pm 38.02 ; p=0.014$ ), interaction force smoothness (difference, $1.43 \pm 0.51 ; p=0.004$ ) and the intent response rate (difference, $0.04 \pm 0.01 ; p=0.008$ ) after

330 the 4-week intervention (see Table 2).

331 Table 2 Exoskeleton kinematic and interaction force metrics

\begin{tabular}{ccccc}
\hline $\begin{array}{c}\text { Kinematic/interaction force } \\
\text { metrics }\end{array}$ & Pre-treatment & Post-treatment & Within-Group Differences & $p$ Value \\
\hline$S_{w}$ & $17.22 \pm 6.99$ & $6.69 \pm 3.49$ & $7.22 \pm 2.55$ & $0.004^{* *}$ \\
$P_{e}$ & $465.33 \pm 128.64$ & $341.87 \pm 171.95$ & $107.54 \pm 38.02$ & $0.014^{*}$ \\
$S_{f}$ & $6.29 \pm 2.70$ & $4.16 \pm 1.82$ & $1.43 \pm 0.51$ & $0.004^{* *}$ \\
$R_{i}$ & $0.17 \pm 0.04$ & $0.22 \pm 0.06$ & $0.04 \pm 0.01$ & $0.008^{* *}$ \\
\hline
\end{tabular}


Overall, the exoskeleton showed high levels of safety and satisfaction (Supplementary material). At 4 weeks, the patients trained showed significant reductions in motor impairment (see Figure 5) and significant improvements in motor capacity and performing activities of living, as measured by the Fugl-Meyer assessment for upper extremities (difference, 6.50 points; 95\% confidence interval [CI], 6.06 to 16.94; $p=0.02$ ), the action research arm test (difference, 3.33 points; $95 \%$ confidence interval [CI], 4.97 to $10.53 ; p=0.003$ ) and the modified Barthel index (difference, 11.22 points; $95 \%$ confidence interval $[\mathrm{CI}], 8.12$ to $26.88 ; p<0.001)$.

\section{Discussion}

We developed a postural synergy-based exoskeleton, Armule, to provide natural human movement training for stroke patients. Armule has five degrees of freedom to provide motion assistance for the shoulder and elbow. Eight stroke patients used this exoskeleton and underwent evaluations. Our preliminary test results showed that in general, Armule was successful in assisting stroke patients with rehabilitation training, and the subjects responded well to the exoskeleton, without adverse reactions related to the exoskeleton. Additionally, the exoskeleton assisted patients in reproducing the human upper limbs' natural movements throughout the tasks. Under the main motor mode, the stroke subjects were able to actively complete functional exercises with the characteristics of natural human movement. 
the following characteristics.

356 1. In the previous works[38, 44], the self-reaching movements of healthy volunteers are analyzed by the PCA method and extracted the above postural synergies, which can account for more than $80 \%$ of the natural movement variation. Since postural synergies of upper limb self-reaching movements were embedded in this exoskeleton's structure, the motions generated by the exoskeleton were in line with the natural movement characteristics of the human upper limb. Recent studies[42, 43] have shown that postural synergy can improve the efficiency of motor learning for healthy volunteers. To our knowledge, this is the first time a postural synergy based rehabilitation exoskeleton robot has been introduced into rehabilitation training for stroke patients[40, 41]. After 4 weeks of the intervention, all subjects showed significant improvements in the clinical outcomes, which indicated that using the postural synergy-based exoskeleton for rehabilitation training is safe and feasible.

2. Our control strategy identified patients' movement intention and facilitated patients to actively participate in functional exercise training by using the admittance control method in the dimension reduction subspace. And unlike previous exoskeleton rehabilitation robots, the incomplete transparent control strategy limited the patients' abnormal synergy movements and encouraged them to perform natural movements. The safety and satisfaction questionnaire showed that the non-transparent control strategy we adopted did not cause patient discomfort. According to the kinematic data analysis, after the 4-week intervention, the stroke 

and maintain the smoothness and similarity of movement as healthy control. It means that abnormal synergy, tremor might be reduced after natural human movement training. In terms of the interaction force with the exoskeleton, the force's smoothness greatly improved, which we speculated was related to the fact that the rehabilitation robot could only complete natural movements following human motion characteristics. The intention response rate results show that our exoskeleton can always perform the corresponding movements in response to the patient's intention to a certain extent (Figure 2). As the rehabilitation progressed, the rate of intention response increased; this suggests a decrease in abnormal synergistic or compensatory motor effects. Although all patients showed improvement in the interaction rate, the absolute magnitude of improvement was small, which may be related to the degree of muscle strength recovery in stroke patients.

3. Our exoskeleton included a mechanical structure and control algorithm that compensated for gravity and friction, significantly improving the equipment's back-drive ability and the patient's ability to participate in training actively. In this way, the whole rehabilitation exoskeleton robot can be driven by only two active

4. We provided visual feedback during training to indicate the direction of the patient's efforts. Patients are encouraged to use a combination of active exercise 
components close to those of healthy to reproduce the natural human movement, which may have helped accelerate motor function restoration in the patients[42].

In this study, there are some limitations worth noting. First, the small sample of participants may limit the generalizability of our findings in terms of efficacy, despite the included patients having statistically significant improvements. Therefore, larger randomized controlled trials that include subjects with heterogeneous characteristics should be conducted to confirm the study results. Second, the relationships between the clinical improvements and robot test indicators need to be more carefully researched and explained. We plan to conduct larger studies to assess the correlations between the robot test index and clinical improvements and adopt motion capture technology and

410 functional magnetic resonance imaging to uncover the evolution of the underlying motor synergies and neural mechanisms of motor performance improvement in stroke patients. Third, this exoskeleton generates motions by a linear combination of postural coordination primitives extracted from 5 self-reaching movements; thus, this exoskeleton only focuses on self-care activities and involves limited movements. In the

415 future, we need to include more movement template libraries to expand the training task types of rehabilitation exoskeletons.

\section{Conclusions}

418 Our preliminary study shows that the subjects are well adapted to our device, assisting 
419 the subjects in completing functional movements with the natural human movement 420 characteristics. The preliminary clinical intervention results indicate that the postural synergy-based exoskeleton improved the subjects' motor control and their upper limbs'

422 ADL function.

\section{Abbreviations}

DOF: Degrees of freedom; FMA-UE: Fugl-Meyer Assessment for upper extremities; ARAT: Action activities of daily living; PCA: Principal component analysis; MMSE: Mini-mental state examination;

Ethics approval and consent to participate

\section{Consent for publication}

The consent for publication had been obtained from all participants.

\section{Availability of data and materials}

435 The datasets generated during the current study are available from the corresponding author on reasonable request. 

relationships that could be construed as a potential conflict of interest.

\section{Funding}

441 This work received financial support for the research and publication of this article from National

442 Natural Science Foundation of China (No. 91648203 and U 1913601).

\section{Authors' contributions}

$444 \mathrm{CH}$ and ZJC designed the study. $\mathrm{CH}$ participated in the selection and development of the data analysis and metrics and has provided approval of the final version to be submitted. ZJC recruited the participants,

446 ran the experiments, collected the data, performed the analysis of the data and participated in manuscript 447 drafting. XLH contributed guidance and advice throughout the process. CHX participated in manuscript 448 drafting and has provided approval of the final version to be submitted. All authors read and approved 449 the final manuscript.

\section{Acknowledgements}

\section{References} and national disability-adjusted life-years (DALYs) for 333 diseases and injuries and healthy life expectancy (HALE) for 195 countries and territories, 1990-2016: a systematic analysis for the Global 
Burden of Disease Study 2016. 2017;390(10100):1260-344.

2. Albert S, Kesselring J. Neurorehabilitation of stroke. J Neurol. 2012;259(5):817-32.

3. Muratori LM, Lamberg EM, Quinn L, Duff SV. Applying principles of motor learning and control to upper extremity rehabilitation. Journal of Hand Therapy. 2013;26(2):94-102.

4. Butefisch CM, Davis BC, Wise SP, Sawaki L, Kopylev L, Classen J, et al. Mechanisms of usedependent plasticity in the human motor cortex. Proc Natl Acad Sci U S A. 2000;97(7):3661-5.

5. Huang VS, Haith A, Mazzoni P, Krakauer JW. Rethinking Motor Learning and Savings in Adaptation Paradigms: Model-Free Memory for Successful Actions Combines with Internal Models. Neuron. 2011;70(4):787-801.

6. Reinkensmeyer DJ, Emken JL, Cramer SC. Robotics, motor learning, and neurologic recovery. Annual Review of Biomedical Engineering. 2004;6:497-525.

7. Coscia M, Wessel MJ, Chaudary U, Millan JDR, Micera S, Guggisberg A, et al. Neurotechnology-aided interventions for upper limb motor rehabilitation in severe chronic stroke. Brain. 2019;142(8):2182-97.

8. Oujamaa L, Relave I, Froger J, Mottet D, Pelissier JY. Rehabilitation of arm function after stroke. Literature review. Ann Phys Rehabil Med. 2009;52(3):269-93.

9. Klamroth-Marganska V, Blanco J, Campen K, Curt A, Dietz V, Ettlin T, et al. Three-dimensional, task-specific robot therapy of the arm after stroke: a multicentre, parallel-group randomised trial. Lancet Neurology. 2014;13(2):159-66.

10. Dimyan MA, Cohen LG. Neuroplasticity in the context of motor rehabilitation after stroke. Nature Reviews Neurology. 2011;7(2):76-85.

11. Lo HS, Xie SQ. Exoskeleton robots for upper-limb rehabilitation: State of the art and future prospects. Medical Engineering \& Physics. 2012;34(3):261-8.

12. Gopura RARC, Bandara DSV, Kiguchi K, Mann GKI. Developments in hardware systems of active upper-limb exoskeleton robots: A review. Robotics and Autonomous Systems. 2016;75:20320.

13. Perry JC, Rosen J, Burns S. Upper-Limb Powered Exoskeleton Design. IEEE/ASME Transactions on Mechatronics. 2007;12(4):408-17.

14. Fabian Just KB, Robert Riener. Online Adaptive Compensation of the ARMin Rehabilitation Robot. BioRob2016.

15. Kim B, Deshpande AD. An upper-body rehabilitation exoskeleton Harmony with an anatomical shoulder mechanism: Design, modeling, control, and performance evaluation. The International Journal of Robotics Research. 2017;36(4):414-35.

16. Carignan C, Tang J, Roderick S, editors. Development of an exoskeleton haptic interface for virtual task training. Intelligent Robots and Systems, 2009 IROS 2009 IEEE/RSJ International Conference on; 2009 10-15 Oct. 2009.

17. Otten A, Voort C, Stienen A, Aarts R, van Asseldonk E, van der Kooij H. LIMPACT: A Hydraulically Powered Self-Aligning Upper Limb Exoskeleton. leee-Asme Transactions on Mechatronics. 2015;20(5):2285-98.

18. Zimmermann Y, Forino A, Riener R, Hutter M. ANYexo: A Versatile and Dynamic Upper-Limb Rehabilitation Robot. IEEE Robotics and Automation Letters. 2019;4(4):3649-56.

19. Shirota C, Jansa J, Diaz J, Balasubramanian S, Mazzoleni S, Borghese NA, et al. On the assessment of coordination between upper extremities: towards a common language between rehabilitation engineers, clinicians and neuroscientists. Journal of Neuroengineering and 
Rehabilitation. 2016;13.

20. Guidali M, Duschau-Wicke A, Broggi S, Klamroth-Marganska V, Nef T, Riener R. A robotic system to train activities of daily living in a virtual environment. Med Biol Eng Comput. 2011;49(10):1213-23.

21. Wong AL, Haith AM, Krakauer JW. Motor Planning. Neuroscientist. 2015;21(4):385-98.

22. Makowski NS, Knutson JS, Chae J, Crago PE. Control of Robotic Assistance Using Poststroke Residual Voluntary Effort. leee Transactions on Neural Systems and Rehabilitation Engineering. 2015;23(2):221-31.

23. Tacchino G, Gandolla M, Coelli S, Barbieri R, Pedrocchi A, Bianchi AM. EEG Analysis During Active and Assisted Repetitive Movements: Evidence for Differences in Neural Engagement. leee Transactions on Neural Systems and Rehabilitation Engineering. 2017;25(6):761-71.

24. Mihara M, Hattori N, Hatakenaka M, Yagura H, Kawano T, Hino T, et al. Near-infrared Spectroscopy-mediated Neurofeedback Enhances Efficacy of Motor Imagery-based Training in Poststroke Victims A Pilot Study. Stroke. 2013;44(4):1091-+.

25. Borton D, Micera S, Millán JdR, Courtine GJStm. Personalized neuroprosthetics. Science Translational Medicine. 2013;5(210):210rv2-rv2.

26. Sawers A, Ting LH. Perspectives on human-human sensorimotor interactions for the design of rehabilitation robots. Journal of Neuroengineering and Rehabilitation. 2014;11.

27. Hogan N, Krebs HI. Interactive robots for neuro-rehabilitation. Restor Neurol Neurosci. 2004;22(3-5):349-58.

28. Jarrasse N, Proietti T, Crocher V, Robertson J, Sahbani A, Morel G, et al. Robotic exoskeletons: a perspective for the rehabilitation of arm coordination in stroke patients. Frontiers in Human Neuroscience. 2014;8.

29. Blank A, French J, Pehlivan A, O'Malley M. Current Trends in Robot-Assisted Upper-Limb Stroke Rehabilitation: Promoting Patient Engagement in Therapy. Curr Phys Med Rehabil Rep. 2014;2(3):184-95.

30. Rehmat N, Zuo J, Meng W, Liu Q, Xie SQ, Liang H. Upper limb rehabilitation using robotic exoskeleton systems: a systematic review. International Journal of Intelligent Robotics and Applications. 2018;2(3):283-95.

31. Profeta VLS, Turvey MT. Bernstein's levels of movement construction: A contemporary perspective. Human Movement Science. 2018;57:111-33.

32. Bruton M, O'Dwyer $\mathrm{N}$. Synergies in coordination: a comprehensive overview of neural, computational, and behavioral approaches. Journal of Neurophysiology. 2018;120(6):2761-74.

33. Santello M, Bianchi M, Gabiccini M, Ricciardi E, Salvietti G, Prattichizzo D, et al. Hand synergies: integration of robotics and neuroscience for understanding the control of biological and artificial hands. Physics of life reviews. 2016;17:1-23.

34. Hashiguchi Y, Ohata K, Kitatani R, Yamakami N, Sakuma K, Osako S, et al. Merging and Fractionation of Muscle Synergy Indicate the Recovery Process in Patients with Hemiplegia: The First Study of Patients after Subacute Stroke. Neural Plasticity. 2016.

35. Sarasola-Sanz A, Irastorza-Landa N, Lopez-Larraz E, Shiman F, Spuler M, Birbaumer N, et al. Design and effectiveness evaluation of mirror myoelectric interfaces: a novel method to restore movement in hemiplegic patients. Sci Rep. 2018;8(1):16688.

36. Nam C, Rong W, Li W, Xie Y, Hu X, Zheng Y. The Effects of Upper-Limb Training Assisted with an Electromyography-Driven Neuromuscular Electrical Stimulation Robotic Hand on Chronic 


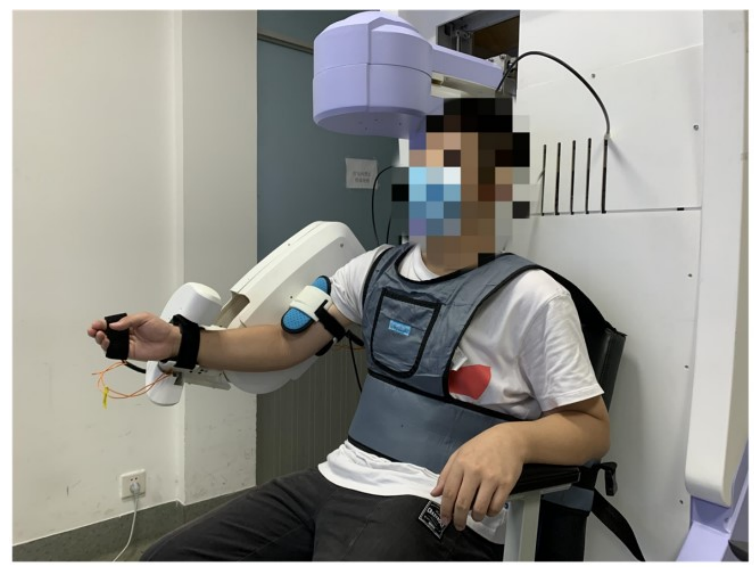

A

Stroke. Front Neurol. 2017;8:679. 6. 2018;99:84-96. Neurorobotics. 2019;13. Biorobotics2017. p. 1175-9. 2017;5(FEB). 2018;15(2):247-59. Rehabilitation. 2011;8.

37. Cheung VCK, Turolla A, Agostini M, Silvoni S, Bennis C, Kasi P, et al. Muscle synergy patterns as physiological markers of motor cortical damage. Proc Natl Acad Sci U S A. 2012;109(36):14652-

38. Liu K, Xiong C-H, He L, Chen W-B, Huang X-L. Postural synergy based design of exoskeleton robot replicating human arm reaching movements. Robotics and Autonomous Systems.

39. Tang S, Chen L, Barsotti M, Hu L, Li Y, Wu X, et al. Kinematic synergy of multi-DOF movement in upper limb and its application for rehabilitation exoskeleton motion planning. Frontiers in

40. Proietti T, Guigon E, Roby-Brami A, Jarrasse N. Modifying upper-limb inter-joint coordination in healthy subjects by training with a robotic exoskeleton. J Neuroeng Rehabil. 2017;14(1):55.

41. Proietti T, Roby-Brami A, Jarrassé N. Learning motor coordination under resistive viscous force fields at the joint level with an upper-limb robotic exoskeleton. Biosystems and

42. Day KA, Bastian AJ. Providing low-dimensional feedback of a high-dimensional movement allows for improved performance of a skilled walking task. Scientific Reports. 2019;9:13.

43. Patel V, Craig J, Schumacher M, Burns MK, Florescu I, Vinjamuri R. Synergy repetition training versus task repetition training in acquiring new skill. Frontiers in Bioengineering and Biotechnology.

44. He L, Xiong C, Liu K, Huang J, He C, Chen W. Mechatronic Design of a Synergetic Upper Limb Exoskeletal Robot and Wrench-based Assistive Control. Journal of Bionic Engineering.

45. Abdullah HA, Tarry C, Lambert C, Barreca S, Allen BO. Results of Clinicians Using a Therapeutic Robotic System in an Inpatient Stroke Rehabilitation Unit. Journal of Neuroengineering and

Figure 1 A: Armule rehabilitation exoskeletal robot. B: Two active joints (red), three coupling joints (black). Pressure sensors and six-dimensional force/torque sensors were installed in the linkage cuffs of the forearm and upper arm, respectively, which were used to estimate the patient's motion intention. 


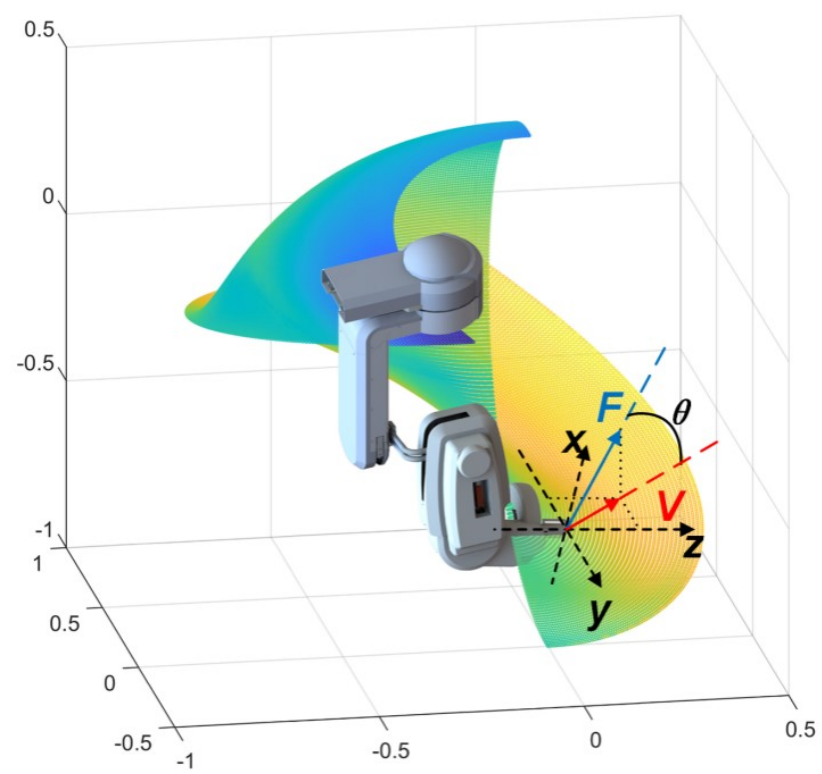

577 Figure 2 The calculated movement intention of the patient might be inconsistent with the actual movement direction of the exoskeleton. The surface represents the workspace of the exoskeleton. $\boldsymbol{F}$ is the equivalent interaction force at the end of the exoskeleton, which was taken as the patient's motion intention. $\boldsymbol{V}$ is the actual velocity vector of exoskeleton in workspace. The Angle between $\boldsymbol{F}$ and $\boldsymbol{V}$ is $\theta$, which will be used for calculating intent response rate later in this article.

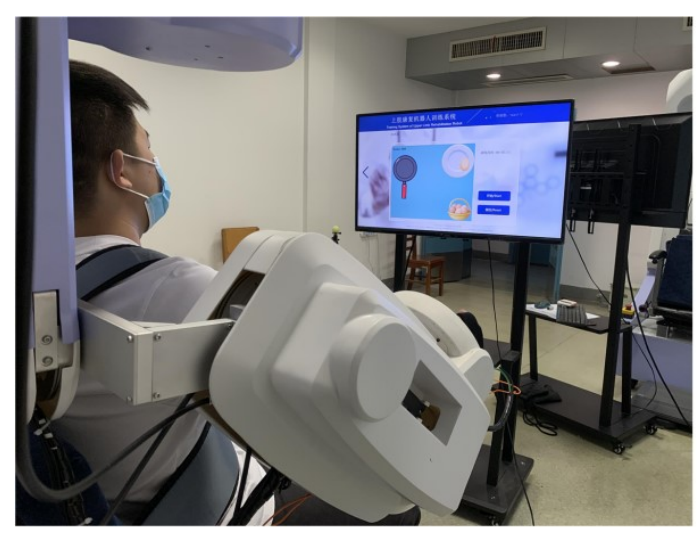

A

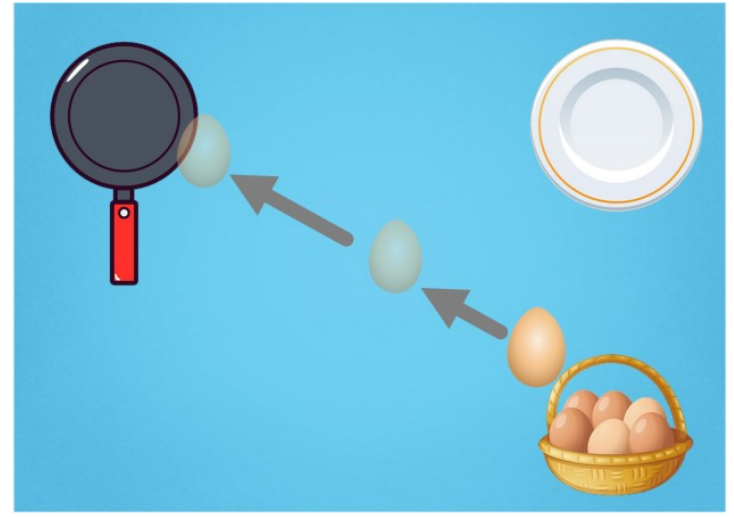

B

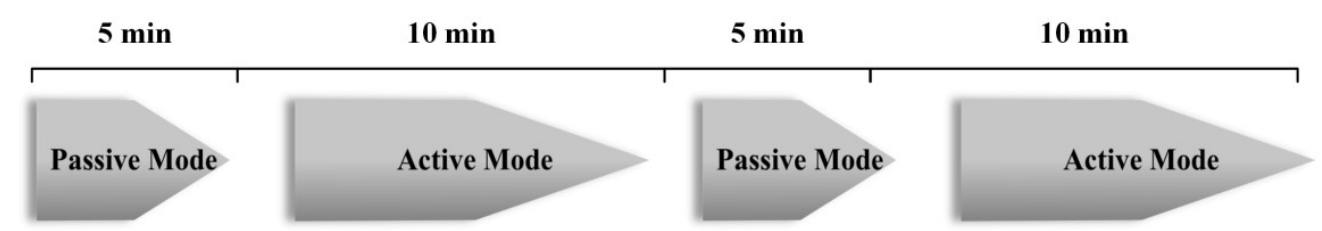

C

Figure $3 \mathrm{~A}$ : The exoskeleton calculates and assists the patient in performing the natural reaching movement. B: The frying eggs task, there is visual feedback during training to indicate the direction of the patient's exercise efforts. The participants were asked to perform two sets of exercises per session. Each set consisted of 5 minutes of passive training and 10 minutes of active training. 

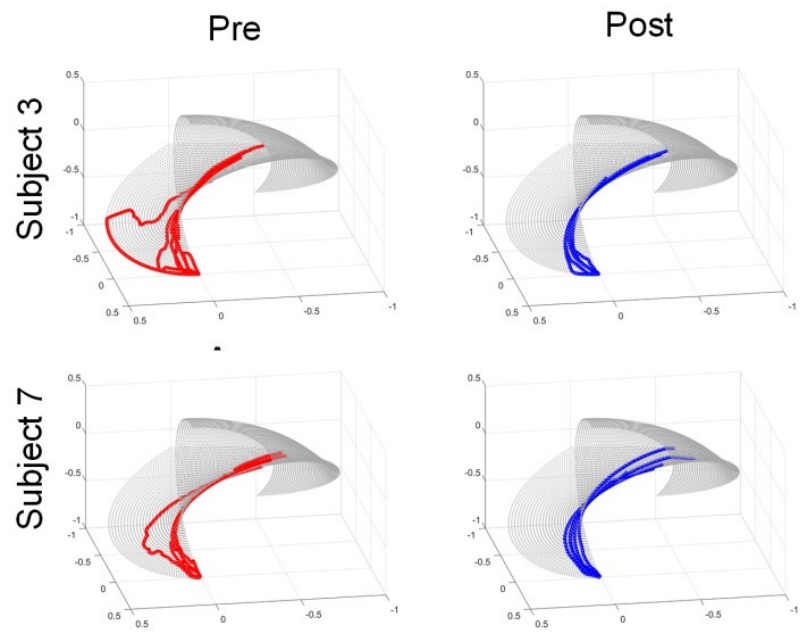

589

the ARAT score and the MBI score.

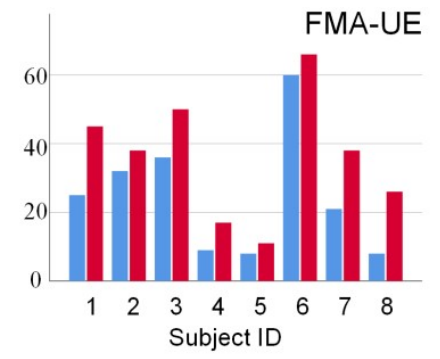

Figure 4 Two exemplar subjects' performance in the reaching tasks pre-treatment and post-treatment. The four panels represent the curves of the exoskeleton end trajectory in workspace during the timenormalized tasks. As shown in the panels, the end trajectory in post-treatment(blue) are smoother and more focused than the end trajectory in pre-treatment(red).
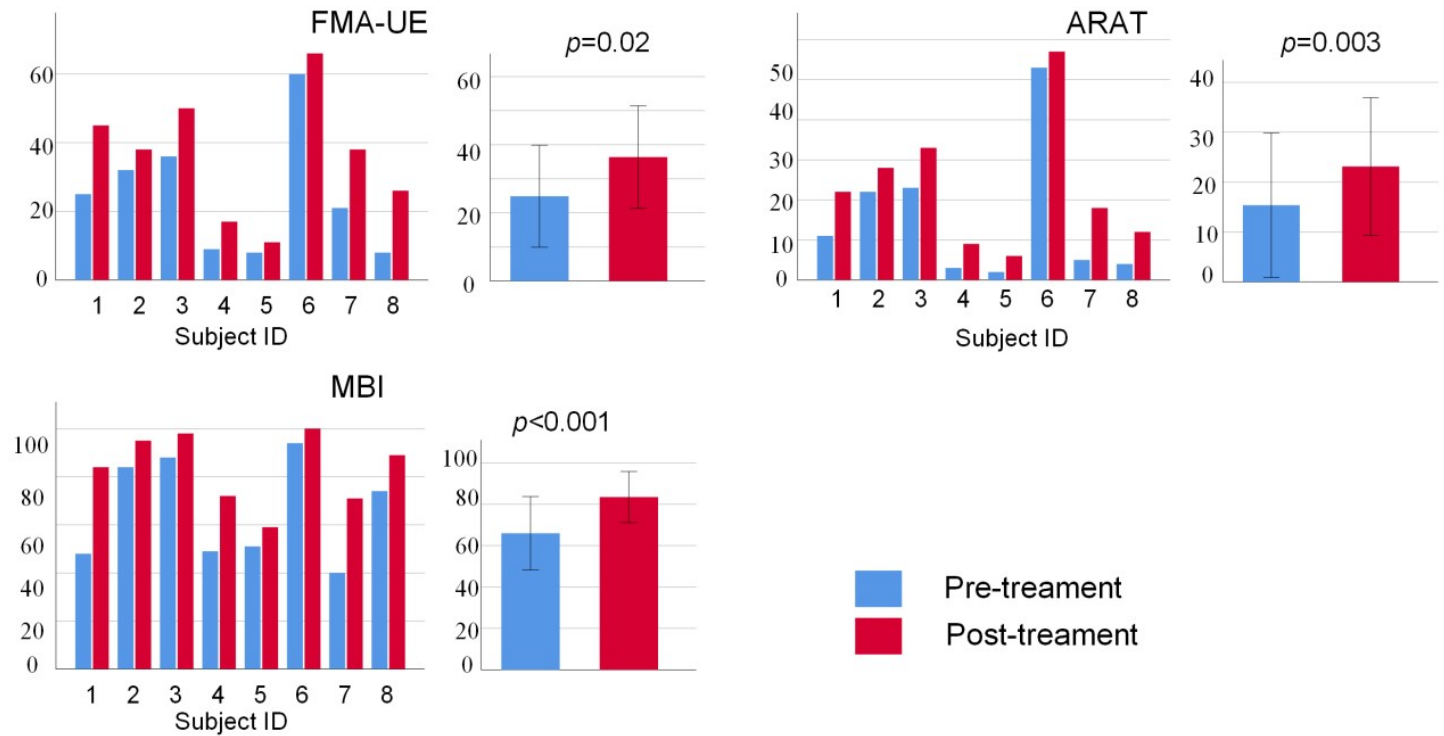


\section{Figures}

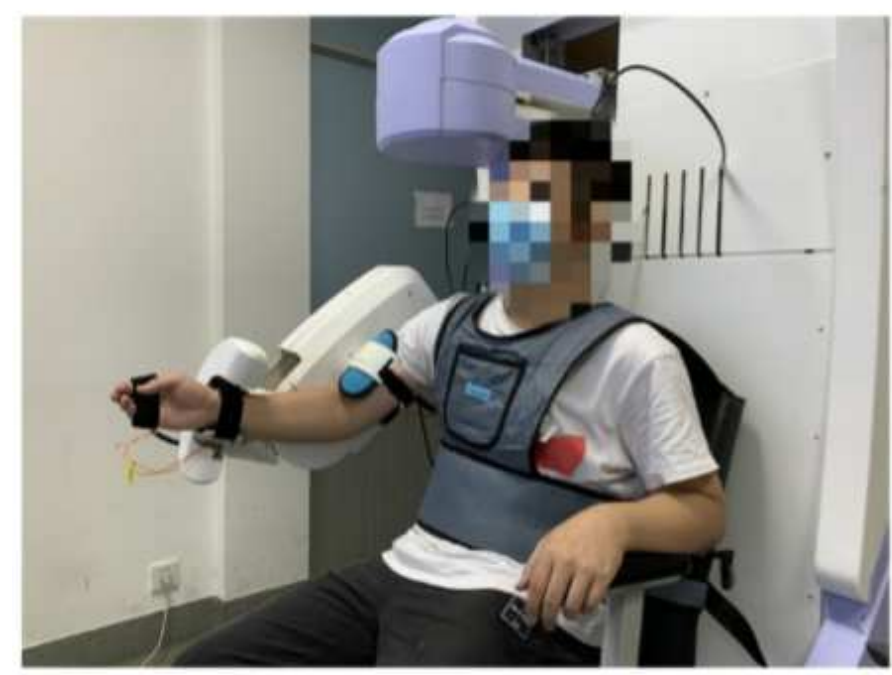

A

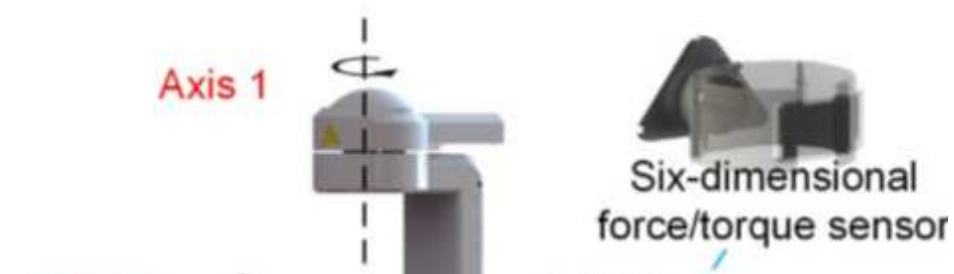

Axis 3

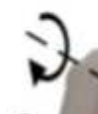

I Axis 2
Axis 4

Axis 5

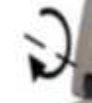

Et

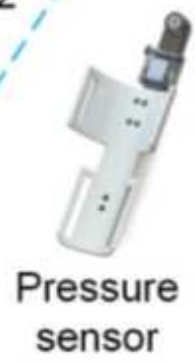

B

Figure 1

A: Armule rehabilitation exoskeletal robot. B: Two active joints (red), three coupling joints (black). Pressure sensors and six-dimensional force/torque sensors were installed in the linkage cuffs of the forearm and upper arm, respectively, which were used to estimate the patient's motion intention.

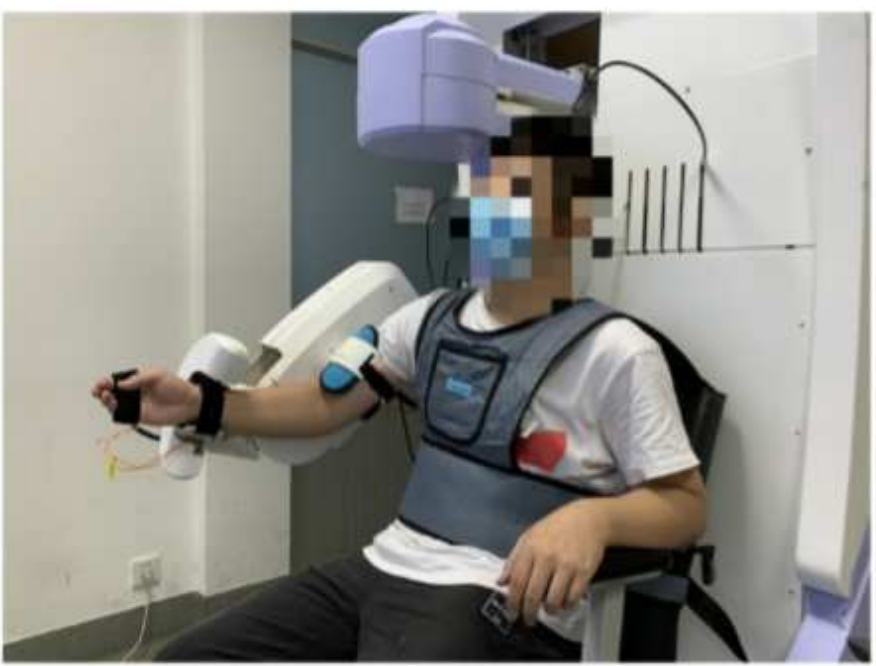

A

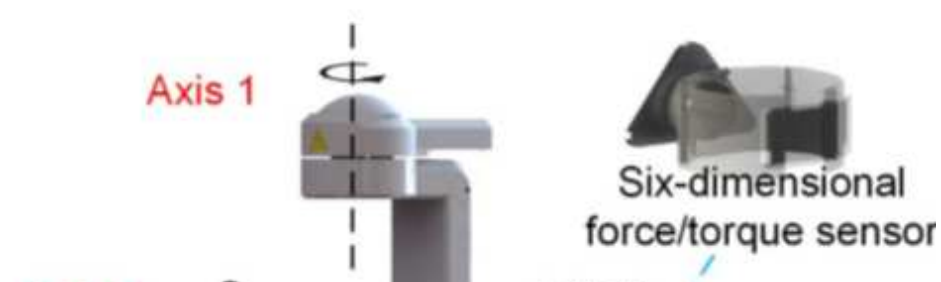

Axis 3 Axis 2

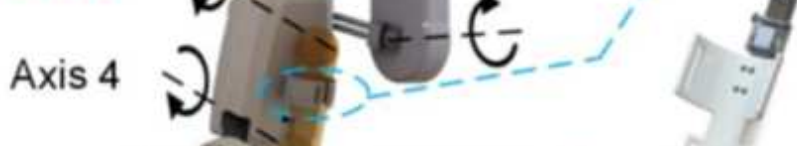

Axis 5

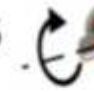

Pressure sensor

B

Figure 1

A: Armule rehabilitation exoskeletal robot. B: Two active joints (red), three coupling joints (black). Pressure sensors and six-dimensional force/torque sensors were installed in the linkage cuffs of the forearm and upper arm, respectively, which were used to estimate the patient's motion intention. 


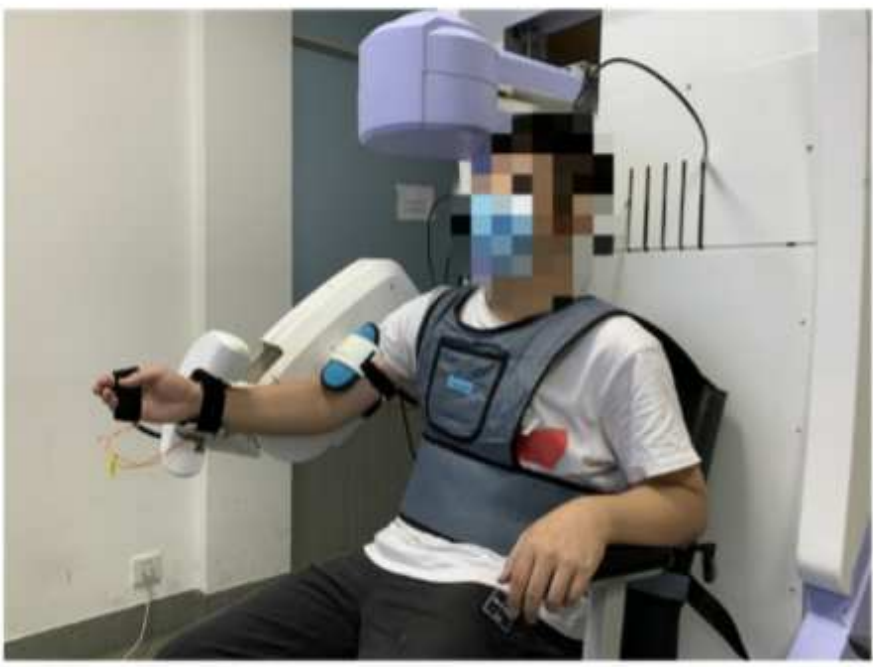

A

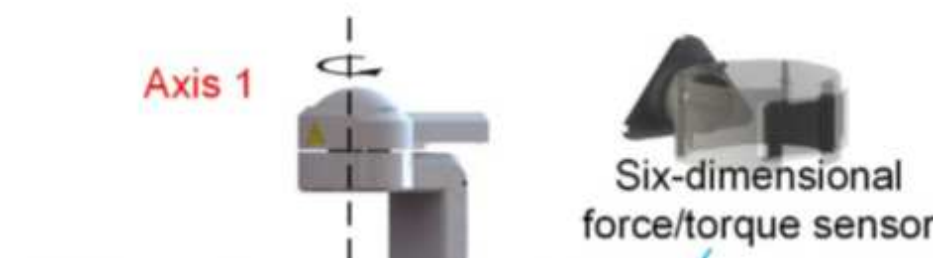

Axis 3

Axis 4

Axis 5

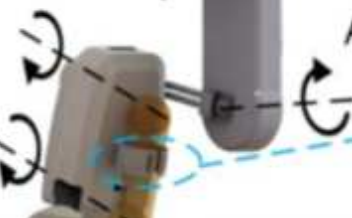
Axis 2

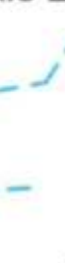

Pressure

sensor

B

\section{Figure 1}

A: Armule rehabilitation exoskeletal robot. B: Two active joints (red), three coupling joints (black). Pressure sensors and six-dimensional force/torque sensors were installed in the linkage cuffs of the forearm and upper arm, respectively, which were used to estimate the patient's motion intention.

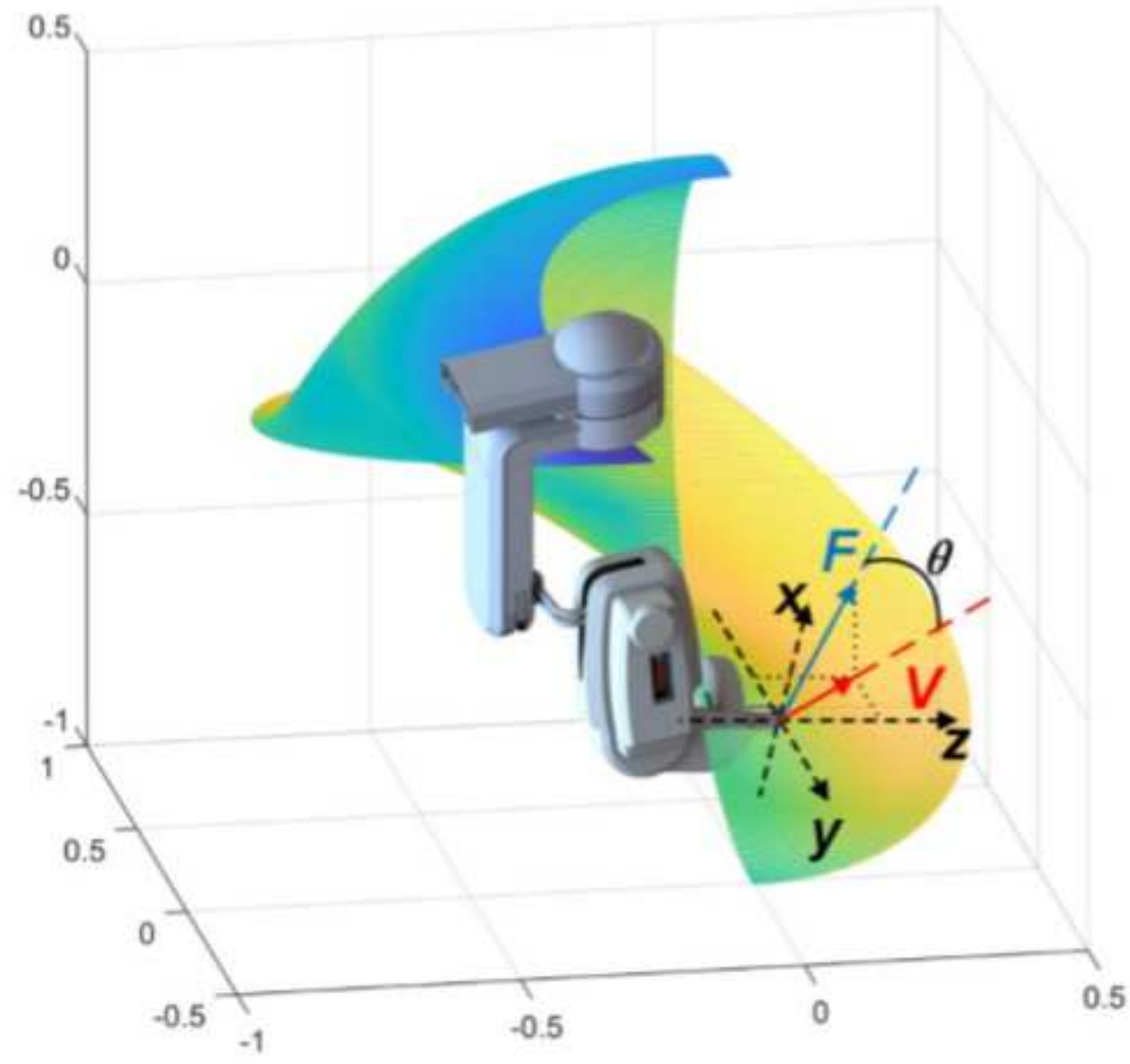

Figure 2 
The calculated movement intention of the patient might be inconsistent with the actual movement direction of the exoskeleton. The surface represents the workspace of the exoskeleton. $\mathrm{F}$ is the equivalent interaction force at the end of the exoskeleton, which was taken as the patient's motion intention. $V$ is the actual velocity vector of exoskeleton in workspace. The Angle between $\mathrm{F}$ and $\mathrm{V}$ is $\mathrm{\nabla}$, which will be used for calculating intent response rate later in this article.

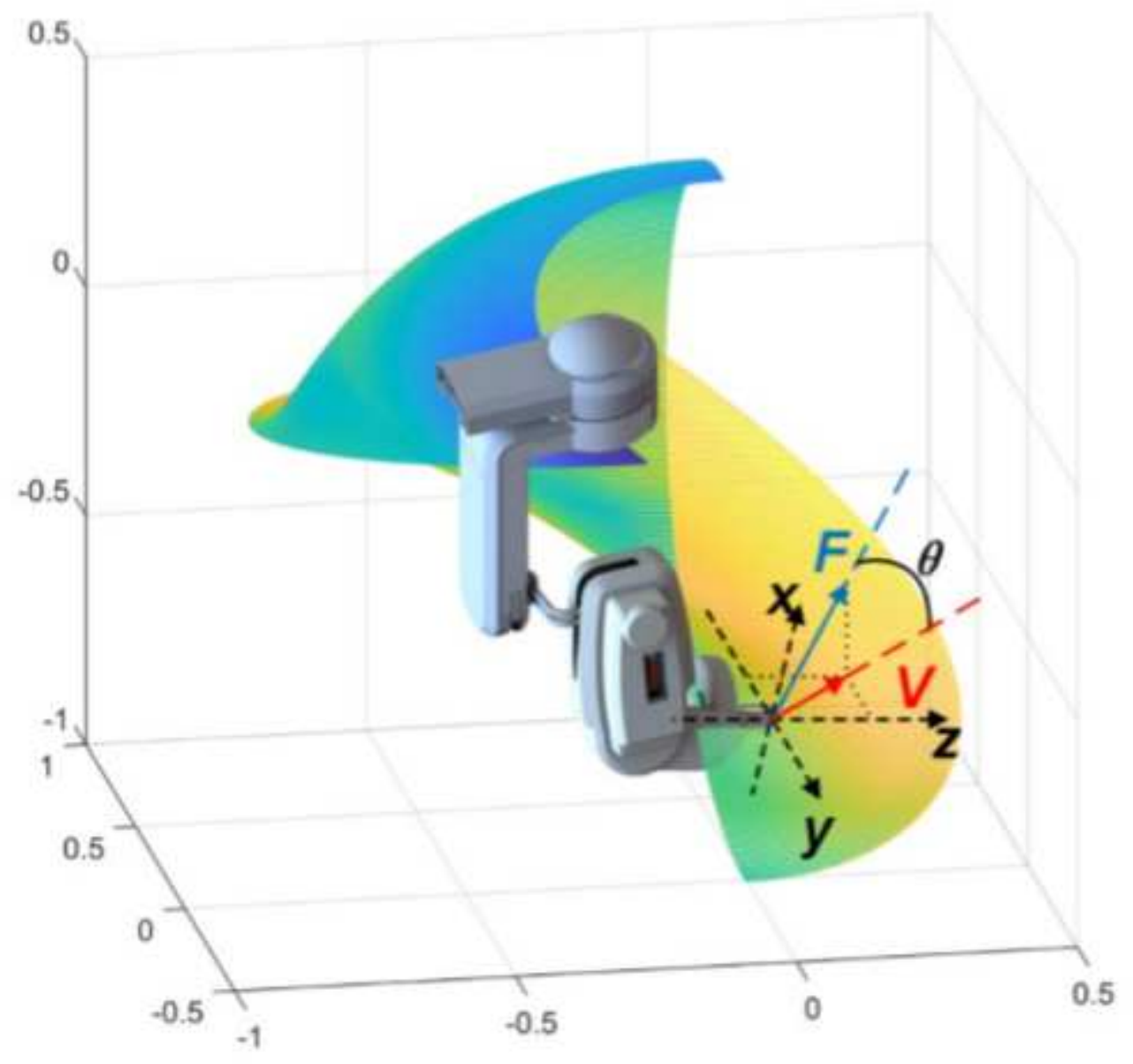

Figure 2

The calculated movement intention of the patient might be inconsistent with the actual movement direction of the exoskeleton. The surface represents the workspace of the exoskeleton. $\mathrm{F}$ is the equivalent interaction force at the end of the exoskeleton, which was taken as the patient's motion intention. $V$ is the actual velocity vector of exoskeleton in workspace. The Angle between $\mathrm{F}$ and $\mathrm{V}$ is $\mathbb{\nabla}$, which will be used for calculating intent response rate later in this article. 


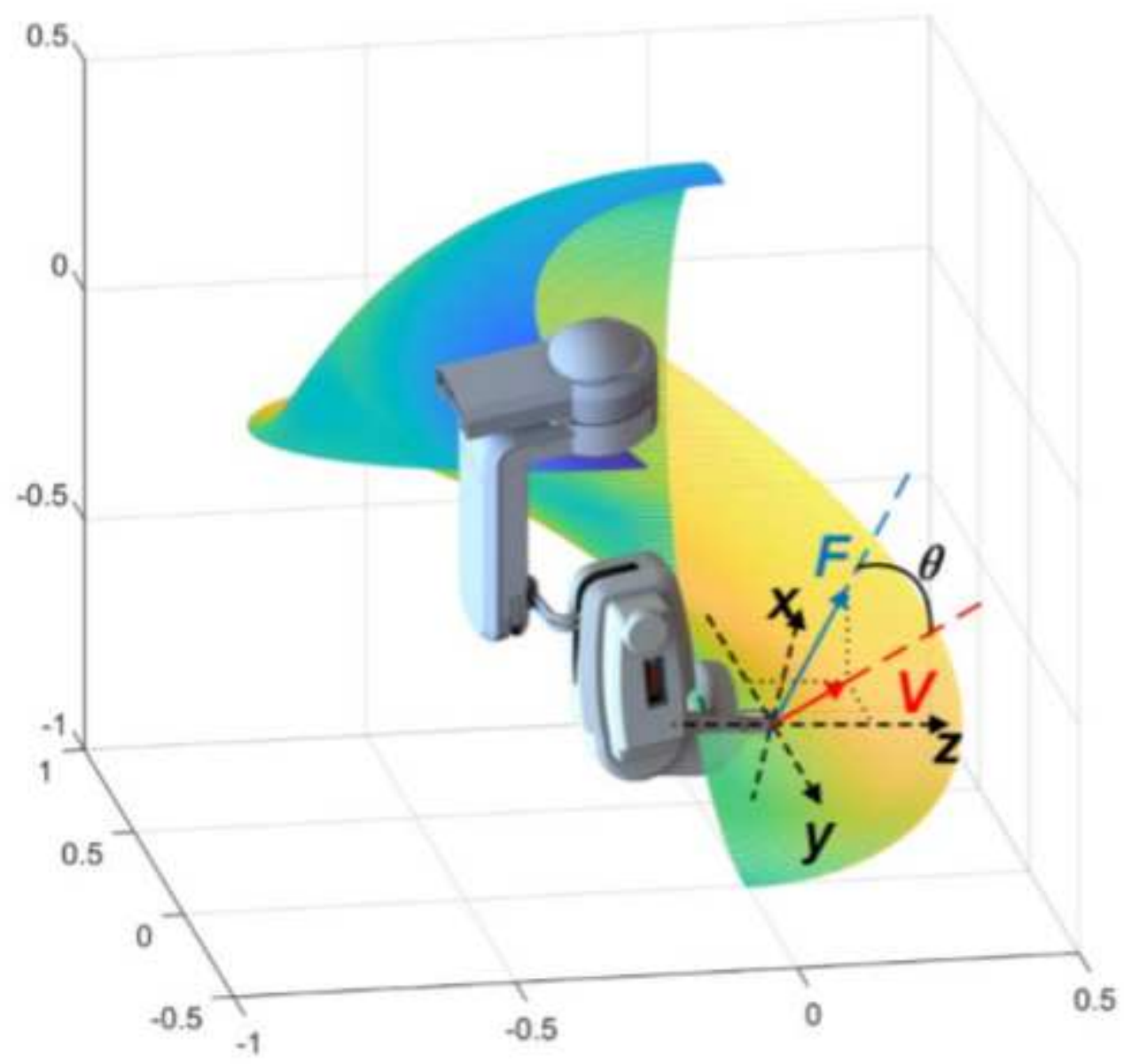

Figure 2

The calculated movement intention of the patient might be inconsistent with the actual movement direction of the exoskeleton. The surface represents the workspace of the exoskeleton. $\mathrm{F}$ is the equivalent interaction force at the end of the exoskeleton, which was taken as the patient's motion intention. $V$ is the actual velocity vector of exoskeleton in workspace. The Angle between $\mathrm{F}$ and $\mathrm{V}$ is $\mathrm{\nabla}$, which will be used for calculating intent response rate later in this article. 


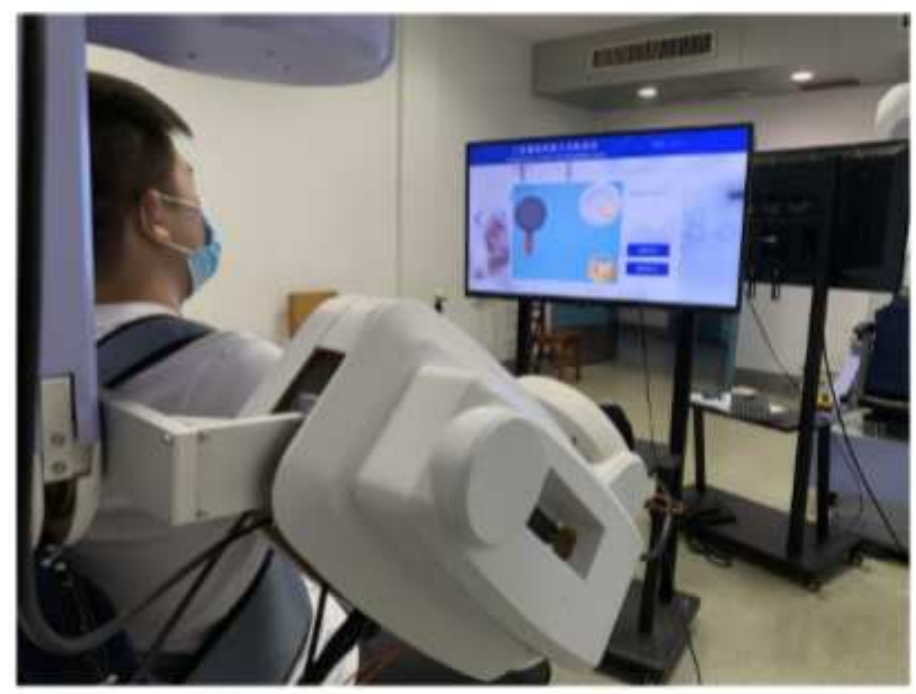

A

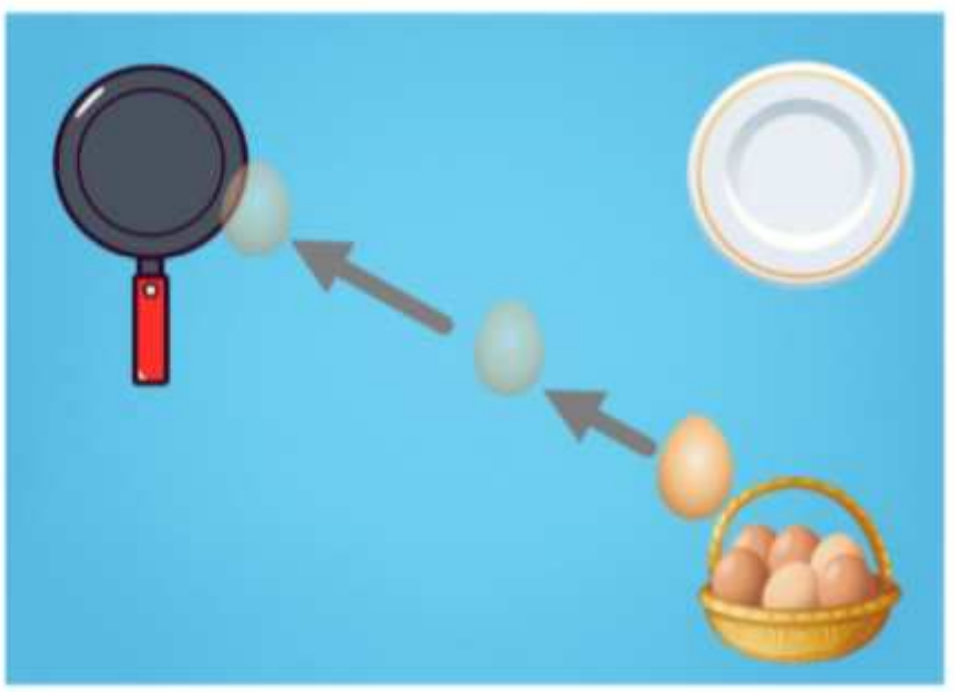

B

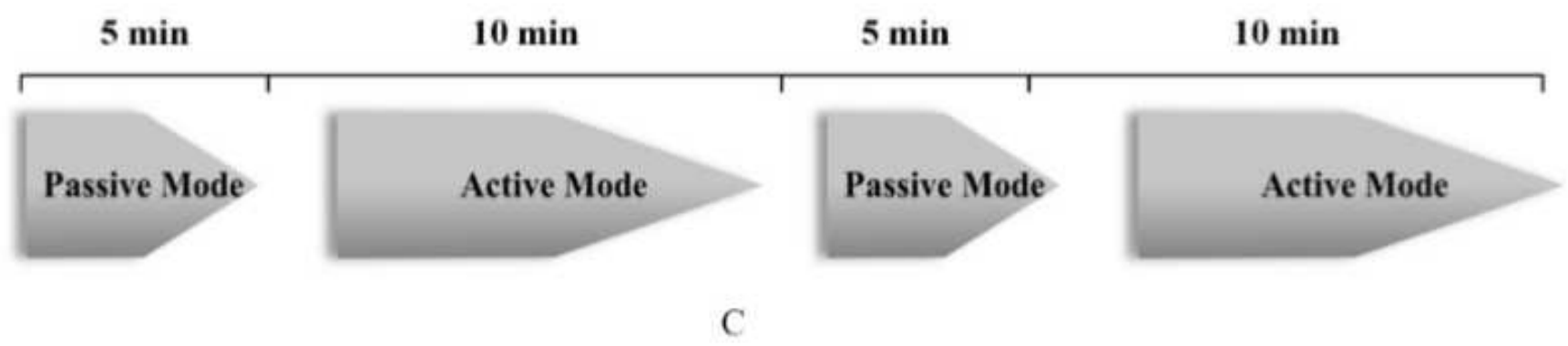

\section{Figure 3}

A: The exoskeleton calculates and assists the patient in performing the natural reaching movement. B: The frying eggs task, there is visual feedback during training to indicate the direction of the patient's exercise efforts. The participants were asked to perform two sets of exercises per session. Each set consisted of 5 minutes of passive training and 10 minutes of active training. 


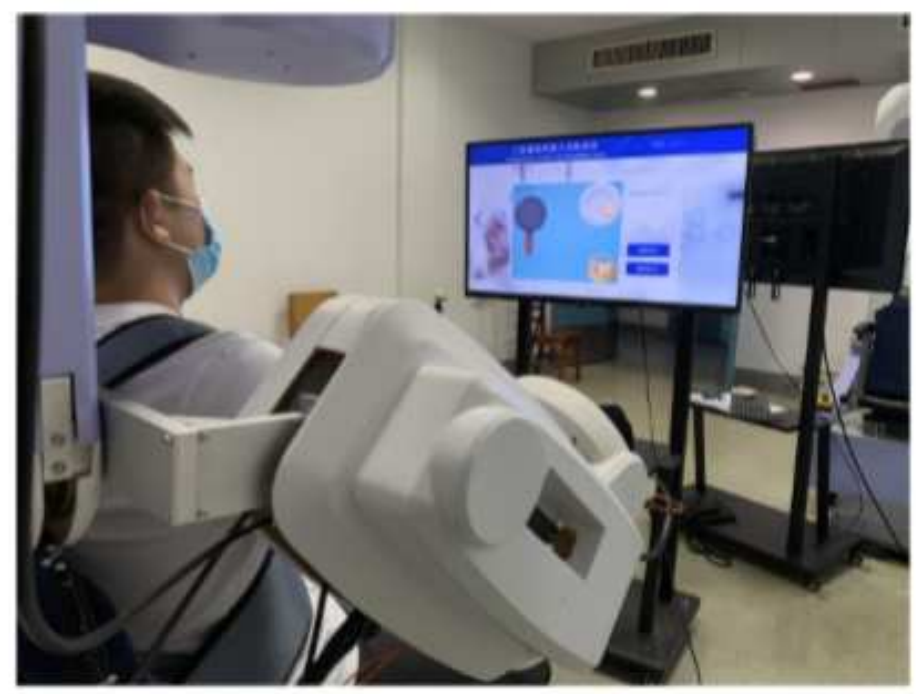

A

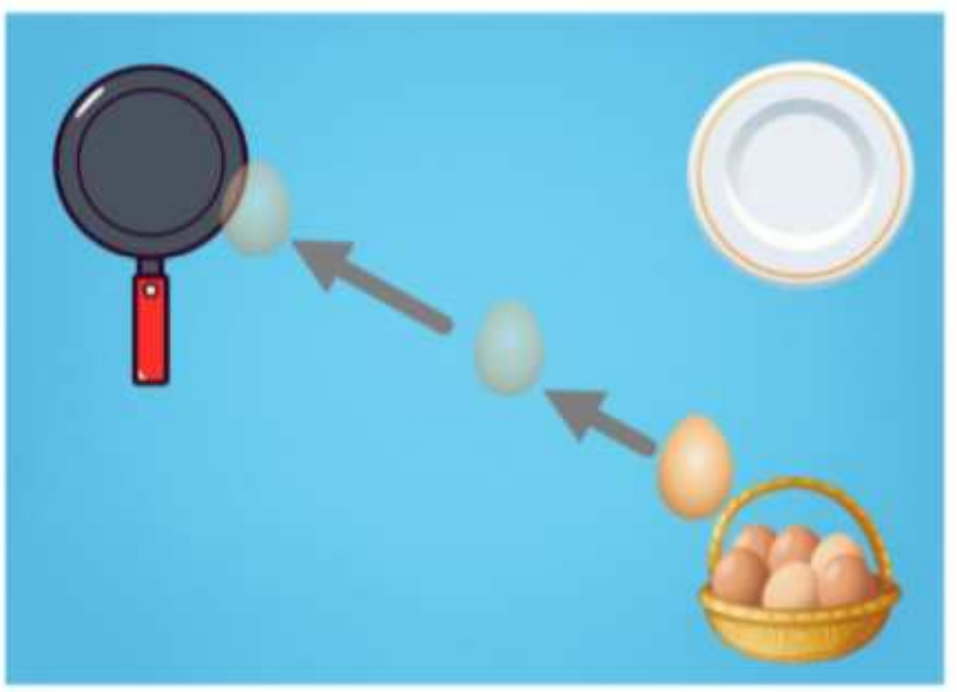

B

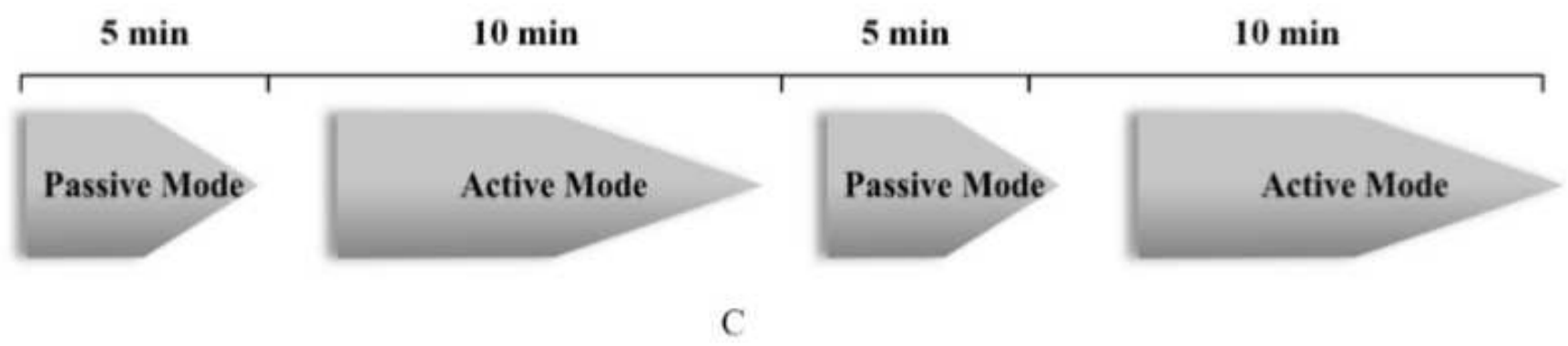

\section{Figure 3}

A: The exoskeleton calculates and assists the patient in performing the natural reaching movement. B: The frying eggs task, there is visual feedback during training to indicate the direction of the patient's exercise efforts. The participants were asked to perform two sets of exercises per session. Each set consisted of 5 minutes of passive training and 10 minutes of active training. 


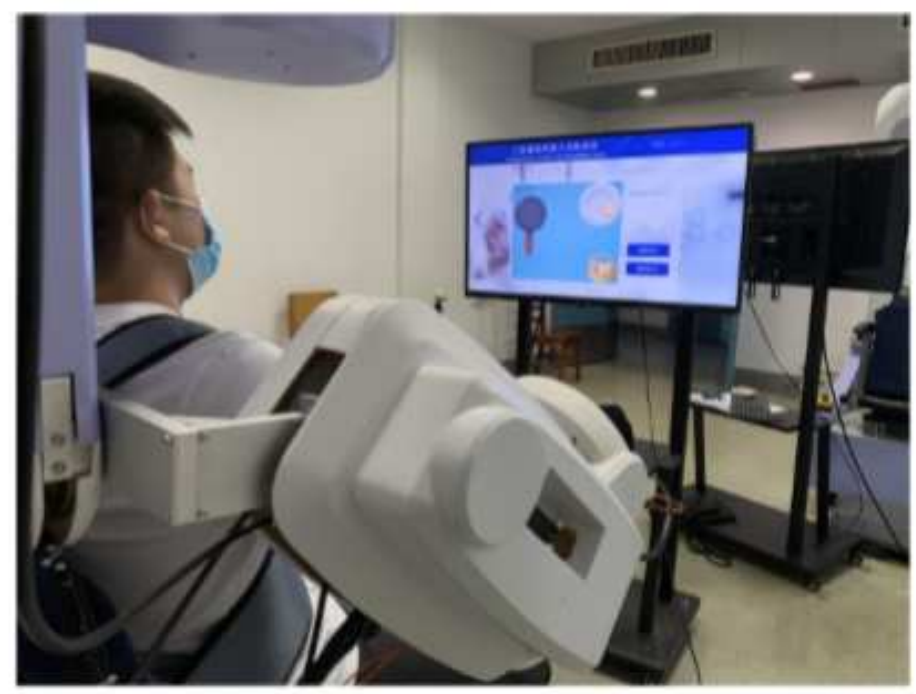

A

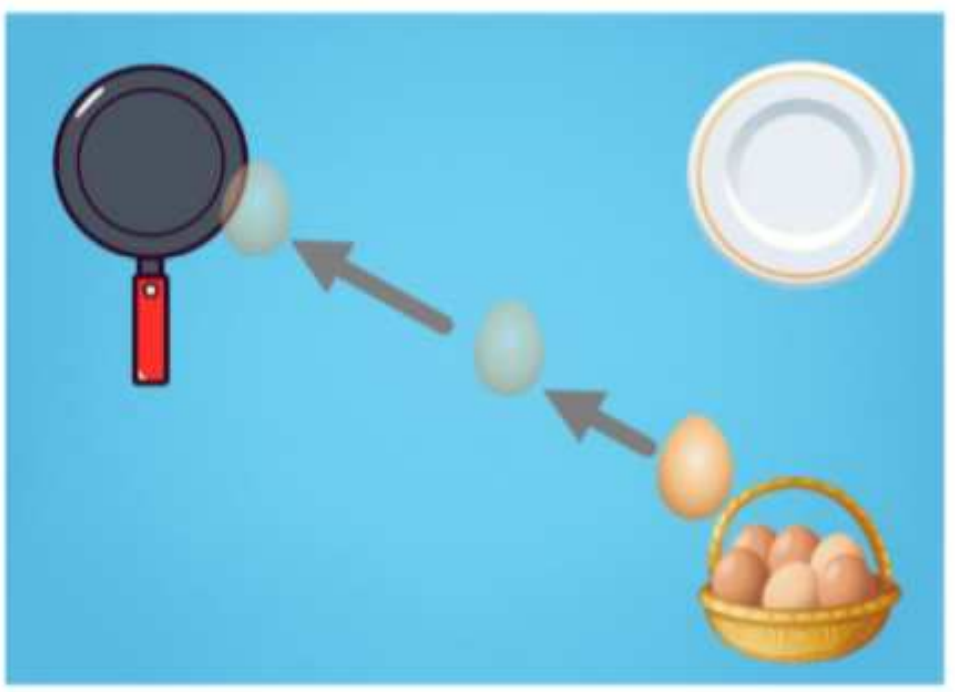

B

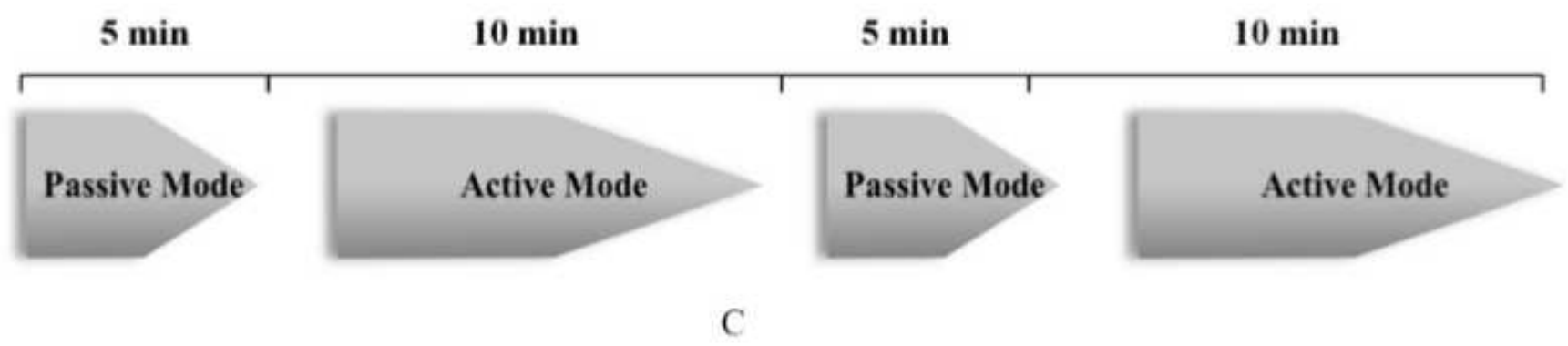

\section{Figure 3}

A: The exoskeleton calculates and assists the patient in performing the natural reaching movement. B: The frying eggs task, there is visual feedback during training to indicate the direction of the patient's exercise efforts. The participants were asked to perform two sets of exercises per session. Each set consisted of 5 minutes of passive training and 10 minutes of active training. 

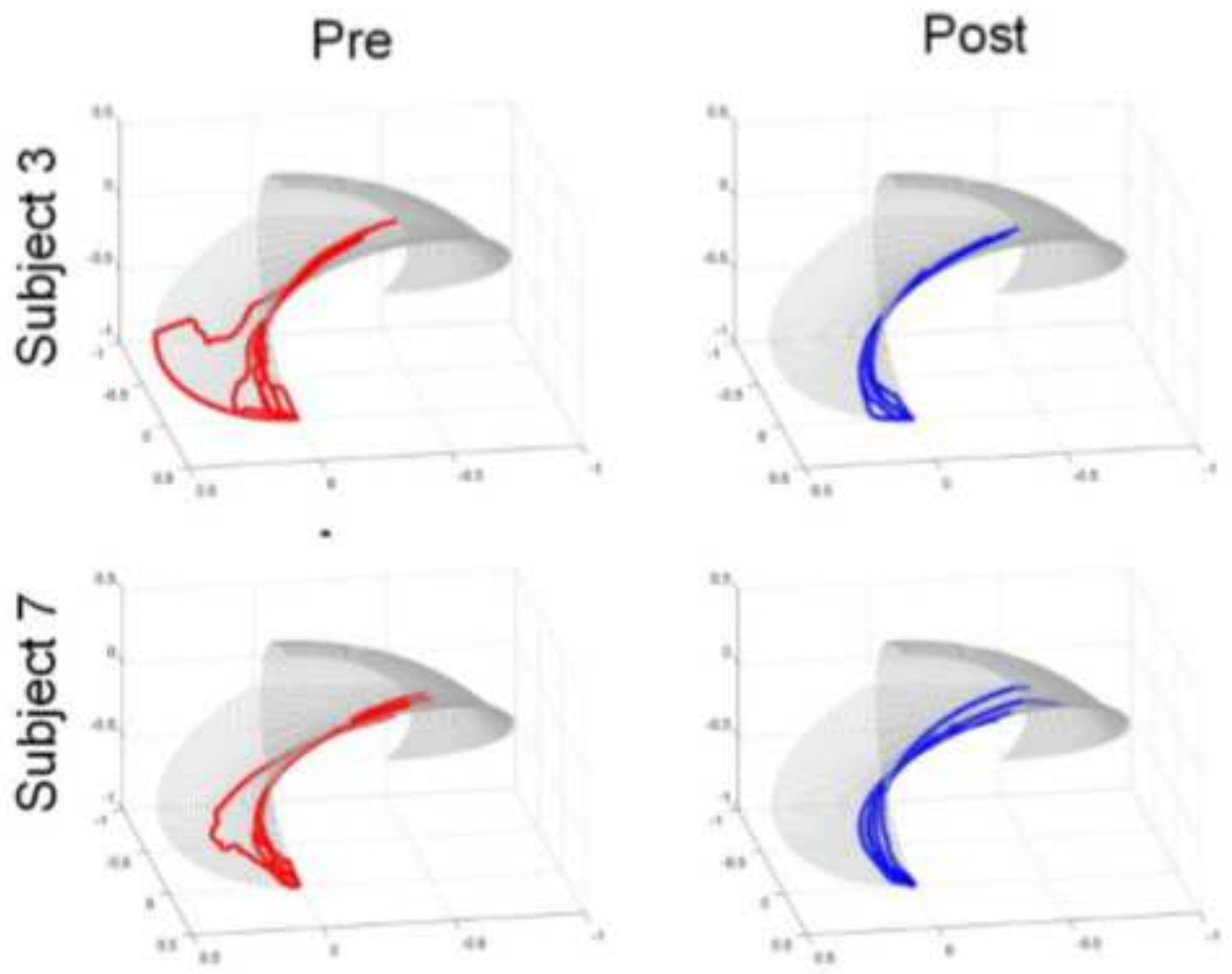

Figure 4

wo exemplar subjects' performance in the reaching tasks pre-treatment and post-treatment. The four panels represent the curves of the exoskeleton end trajectory in workspace during the time-normalized tasks. As shown in the panels, the end trajectory in post-treatment(blue) are smoother and more focused than the end trajectory in pre-treatment(red).

Pre
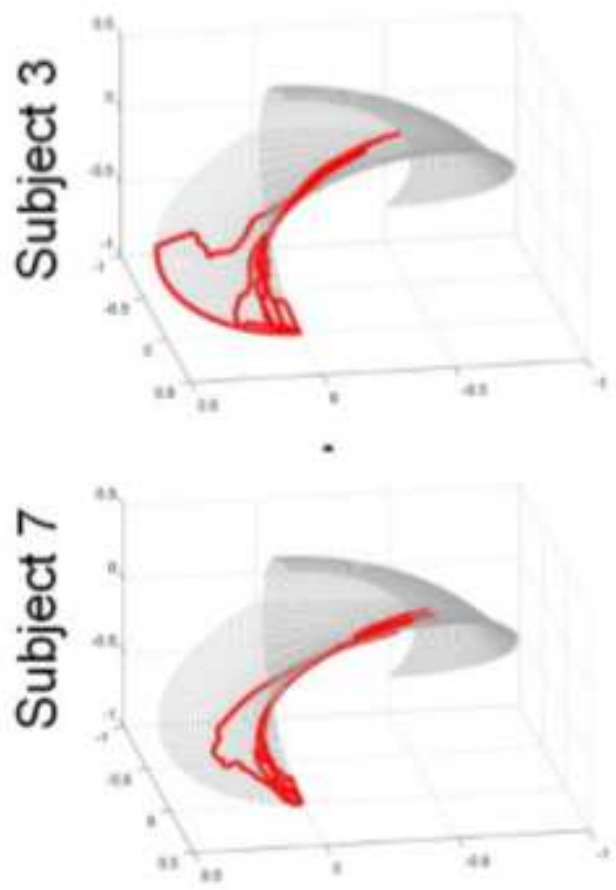

Post
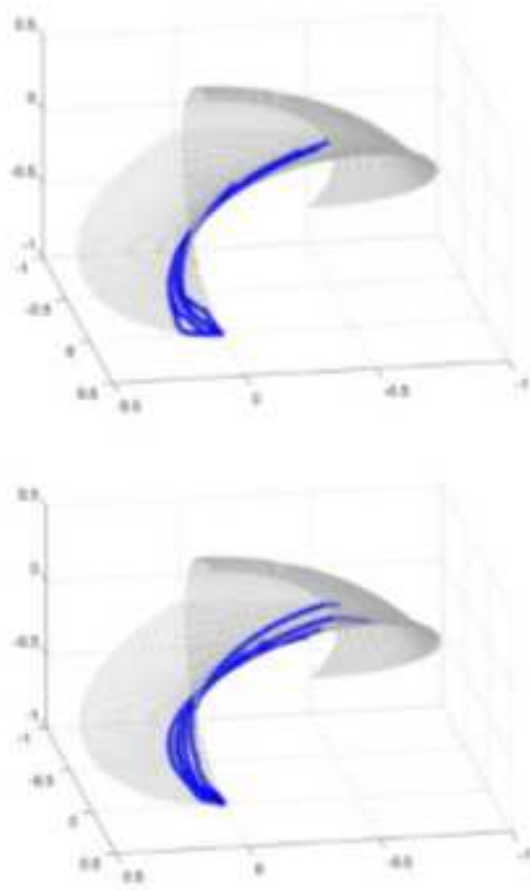


\section{Figure 4}

wo exemplar subjects' performance in the reaching tasks pre-treatment and post-treatment. The four panels represent the curves of the exoskeleton end trajectory in workspace during the time-normalized tasks. As shown in the panels, the end trajectory in post-treatment(blue) are smoother and more focused than the end trajectory in pre-treatment(red).

Pre
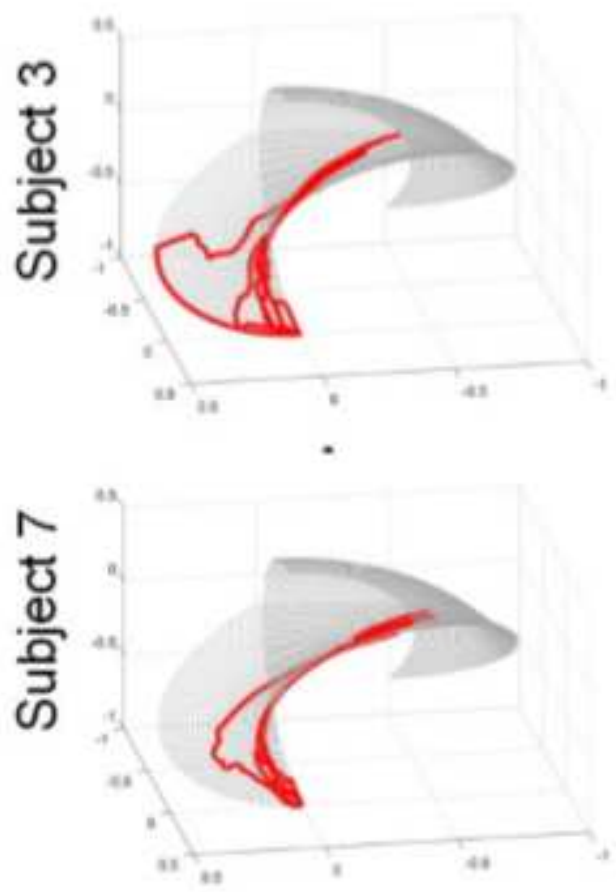

Post
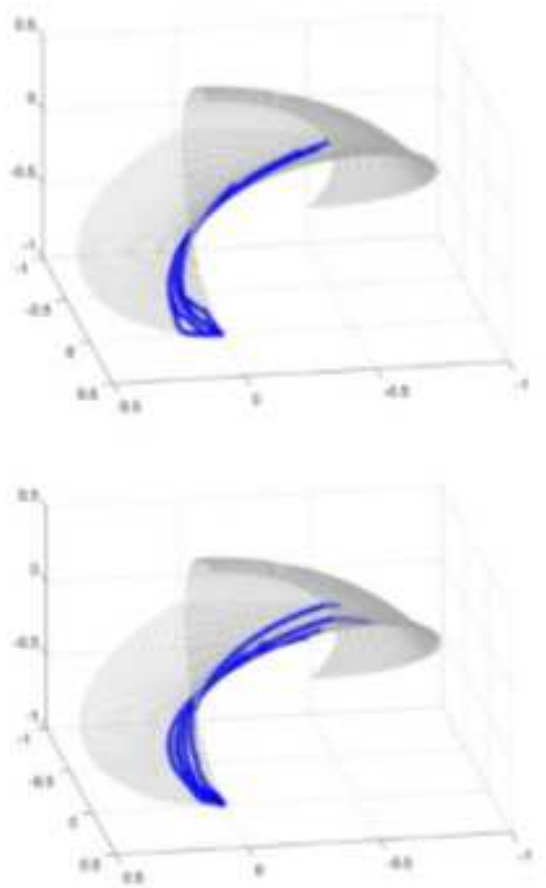

\section{Figure 4}

wo exemplar subjects' performance in the reaching tasks pre-treatment and post-treatment. The four panels represent the curves of the exoskeleton end trajectory in workspace during the time-normalized tasks. As shown in the panels, the end trajectory in post-treatment(blue) are smoother and more focused than the end trajectory in pre-treatment(red). 

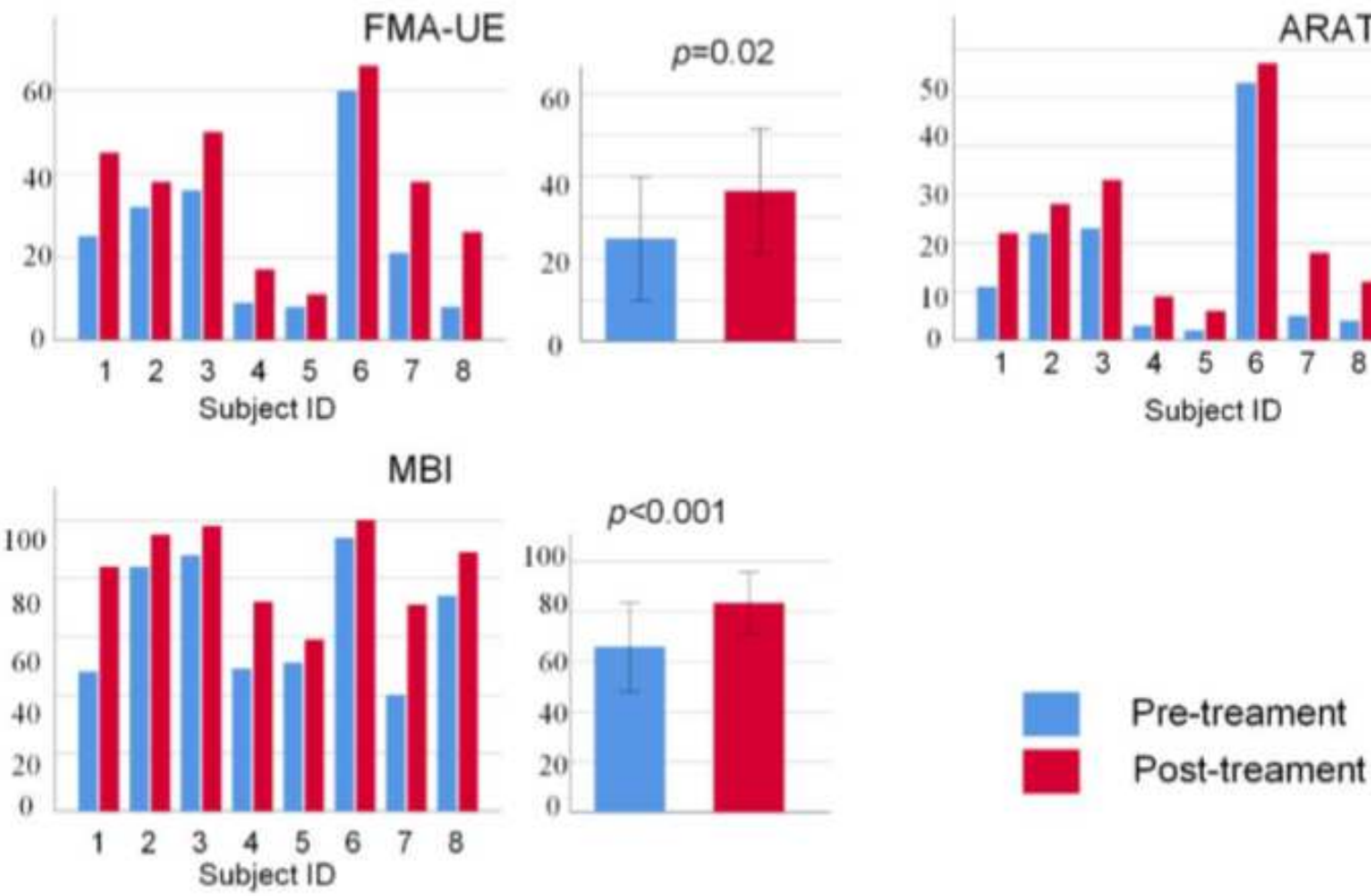

Figure 5

Clinical outcomes, the primary outcome was the FMA-UE score, the secondary outcomes were the ARAT score and the MBI score.
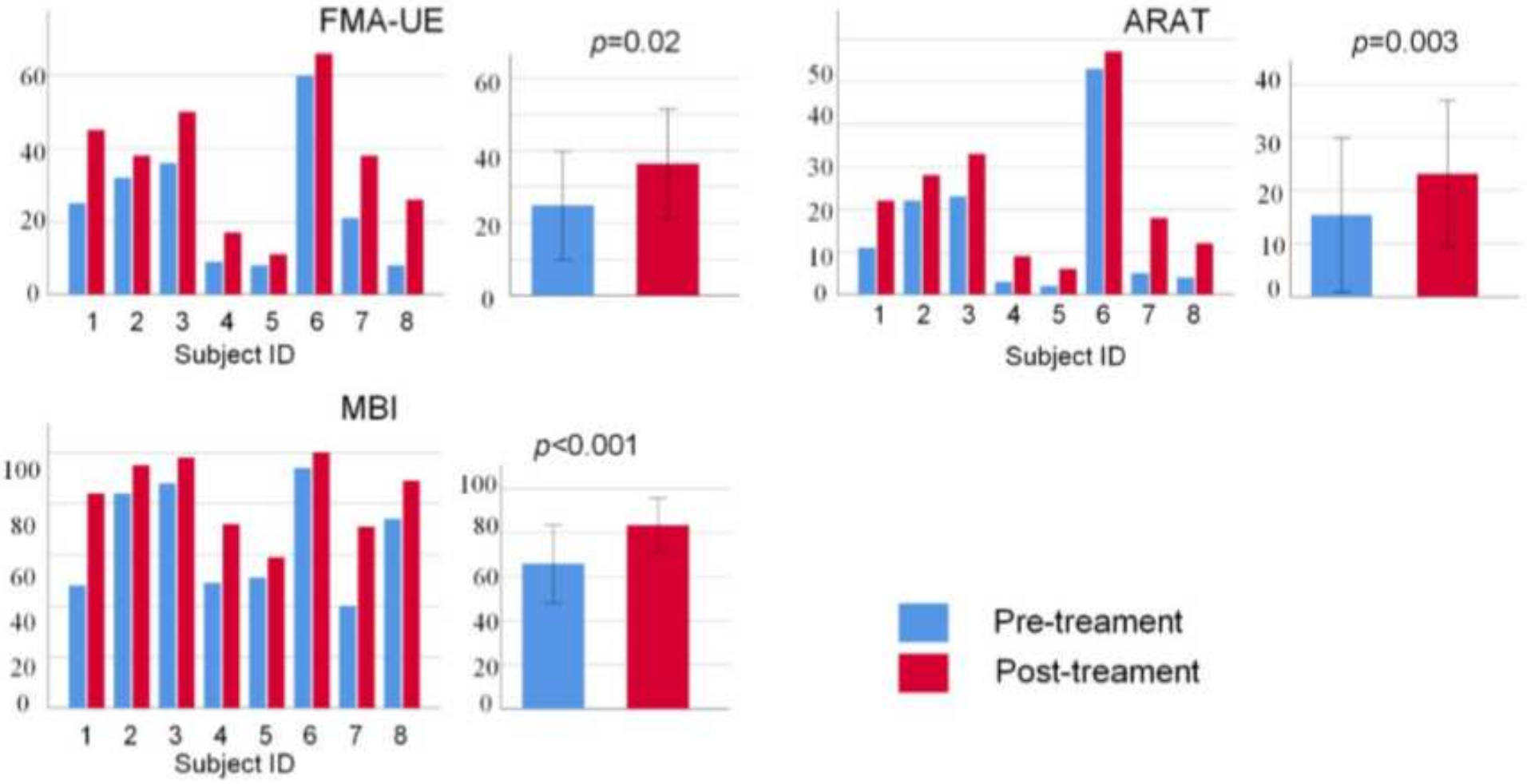

Pre-treament

Post-treament

Figure 5 
Clinical outcomes, the primary outcome was the FMA-UE score, the secondary outcomes were the ARAT score and the $\mathrm{MBI}$ score.
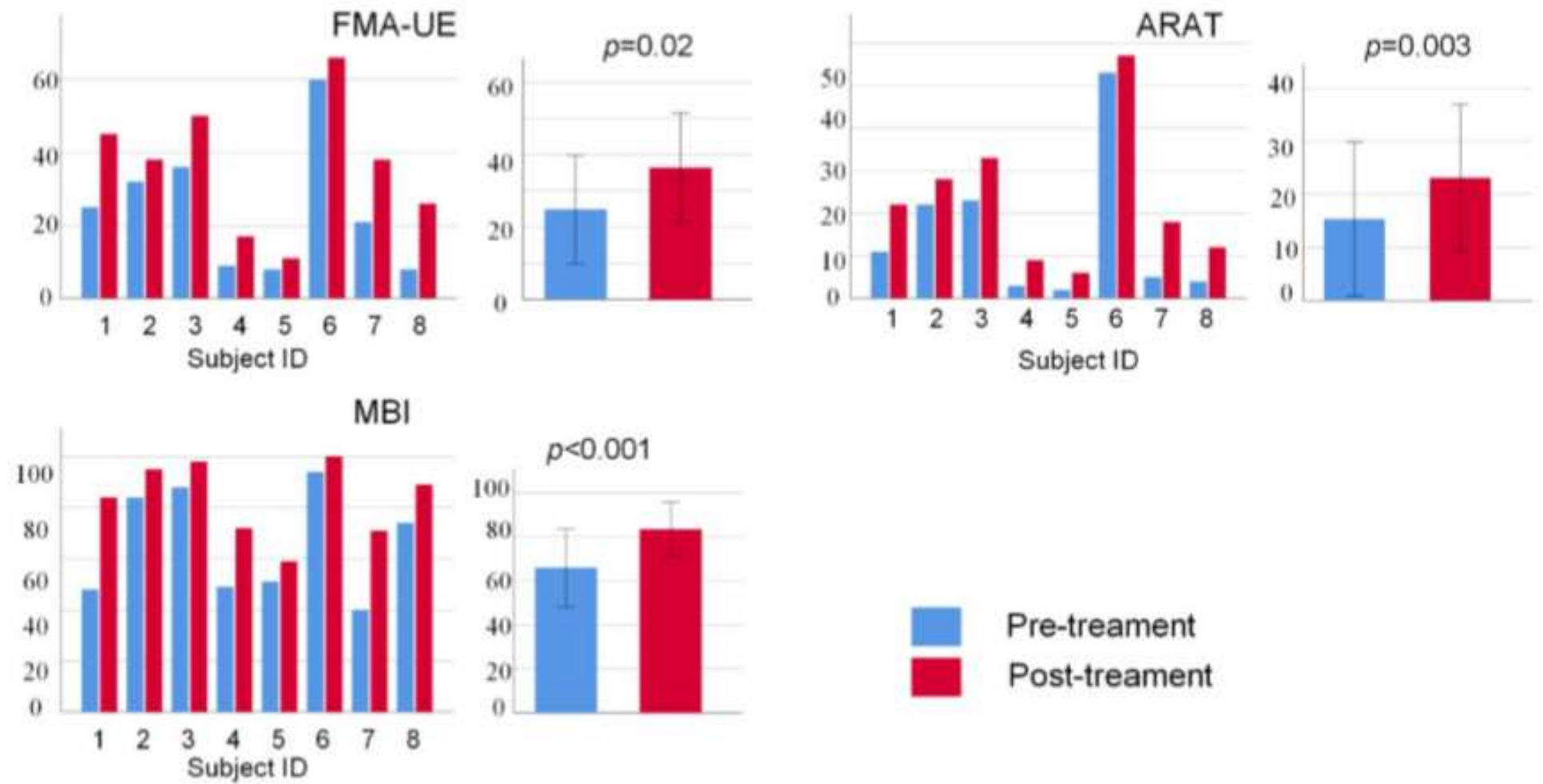

Figure 5

Clinical outcomes, the primary outcome was the FMA-UE score, the secondary outcomes were the ARAT score and the $\mathrm{MBI}$ score.

\section{Supplementary Files}

This is a list of supplementary files associated with this preprint. Click to download.

- SupplementaryMaterial.pdf

- SupplementaryMaterial.pdf

- SupplementaryMaterial.pdf 